\title{
Adaptive Failure Compensation for Aircraft Flight Control Using Engine Differentials: Regulation
}

\author{
Yu Liu, Xidong Tang, and Gang Tao ${ }^{\dagger}$ \\ Department of Electrical and Computer Engineering \\ University of Virginia, Charlottesville, VA 22903 \\ Suresh M. Joshi ${ }^{\ddagger}$ \\ Mail Stop 308, NASA Langley Research Center, Hampton, VA 23681
}

\begin{abstract}
The problem of using engine thrust differentials to compensate for rudder and aileron failures in aircraft flight control is addressed in this paper in a new framework. A nonlinear aircraft model that incorporates engine differentials in the dynamic equations is employed and linearized to describe the aircraft's longitudinal and lateral motion. In this model two engine thrusts of an aircraft can be adjusted independently so as to provide the control flexibility for rudder or aileron failure compensation. A direct adaptive compensation scheme for asymptotic regulation is developed to handle uncertain actuator failures in the linearized system. A design condition is specified to characterize the system redundancy needed for failure compensation. The adaptive regulation control scheme is applied to the linearized model of a large transport aircraft in which the longitudinal and lateral motions are coupled as the result of using engine thrust differentials. Simulation results are presented to demonstrate the effectiveness of the adaptive compensation scheme.
\end{abstract}

Keywords: Actuator failures, adaptive compensation, aircraft model, engine thrust differentials, linearization, stability.

\section{Introduction}

$\mathrm{O}$ July 19, 1989, United Airlines Flight 232, a McDonnell-Douglas DC-10, encountered a terrible disaster during its flight from Denver to Chicago. ${ }^{1,2}$ While flying at a cruise altitude of 37000 feet, the tailmounted engine suffered a catastrophic failure. The fan disk of this engine fragmented and the debris severed all the hydraulic lines, making all three hydraulic flight control systems paralyzed. The pilots controlled the plane by adjusting the thrust differentials of the two wing-mounted engines and managed to reach the runway at Sioux City Airport, Iowa. Unfortunately, the landing was not successful, leading to a broken aircraft and a number of casualties among the passengers. This event demonstrated that even in the presence of severe failures there may still be usable actuation capacity for certain control tasks, and that it is desirable to develop flight control systems that are able to accomplish the desired control tasks when some of aircraft control surfaces fail during the flight. The Flight 232 accident motivated NASA's effort to develop flight control systems that can utilize engine thrusts to maneuver an aircraft when other control surfaces are lost. ${ }^{3}$ In 4, a propulsion-controlled aircraft (PCA) emergency backup control system using only engine thrust for longitudinal control is addressed and flight tested on an MD-11 airplane. In 5, the flight test results of the PCA system on an MD-11 for both longitudinal and lateral control are illustrated. Robust control theory has also been studied for control of aircraft using engine thrusts only. In 6, an H-infinity controller is presented for lateral motion of crippled airplanes and a control strategy is simulated for a Lockheed L-1011 airplane.

Our goal is to develop adaptive control systems that are capable of utilizing the remaining system controls to achieve desired performance in the presence of uncertain actuator failures such as failure of the

\footnotetext{
${ }^{*}$ Graduate Student, Department of Electrical and Computer Engineering, University of Virginia, Charlottesville, VA, 22903

${ }^{\dagger}$ Associate Professor, Department of Electrical and Computer Engineering, University of Virginia, Charlottesville, VA, 22903

${ }_{\ddagger}^{\ddagger}$ Senior Scientist, NASA Langley Research Center, Mail Stop 308, Hampton, Virginia, 23681, Fellow, AIAA.
} 
aircraft rudder system. In this paper, we address some major issues in developing such adaptive failure compensation schemes for aircraft flight control. An engine differential based design is used for rudder or aileron failure compensation. The key issue is to develop an adaptive failure compensation controller which is capable of achieving desired system performance in the presence of uncertain rudder or aileron failures. For this purpose it is necessary to use an aircraft model that incorporates engine differentials in the dynamic equations describing the aircraft's longitudinal and lateral motions. One unique feature of this adaptive failure compensation design is that the controller does not need to know that the failure has occurred, that is, the same controller design works for both normal and failure conditions.

The paper is organized as follows. In Section II, we describe an aircraft dynamic model with engine differentials, in which the two engine thrusts can be adjusted independently. This model provides the needed flexibility for the design of control signals for actuator failure compensation such as rudder failure or engine malfunction. In Section III, we develop an adaptive actuator failure compensation scheme which is able to asymptotically stabilize a class of linear dynamic systems, in the presence of unknown actuator failures. In Section IV, we apply the adaptive failure compensation and stabilization scheme to adaptive rudder and aileron failures compensation using engine thrust differentials, based on the aircraft model which includes the effect of engine differentials. We also present simulation results for a linearized large transport aircraft model with rudder and aileron failures to illustrate the effectiveness of the adaptive failure compensation scheme.

\section{Aircraft Modeling with Engine Differentials}

For the control design and simulation of modern aircraft, a six degree-of-freedom model is usually used in the literature. Such a model is based on the assumption that the engine propulsive forces are symmetric. However, an aircraft model with symmetric engine thrusts is not sufficient for failure compensation control. To compensate aircraft failures such as rudder failure or engine malfunction, an aircraft model with independently adjusted engine thrusts is necessary. In this section, we will first describe an aircraft nonlinear model with independent engine thrusts as well as independent left and right ailerons. A linearized dynamic model will be described at the end of this section. Unlike models based on symmetric engine thrusts, the aircraft models with non-symmetric engine thrusts cannot be decoupled into separate longitudinal and lateral motion equations.

\section{A. Nonlinear Aircraft Model}

Before introducing an aircraft model with engine thrust differentials, we recall that under the assumption of geometric and mass symmetry about the body axis $x-z$ plane in the NASA standard coordinate system, as given in references 7,8 , and 9 , the nonlinear rigid body force and moment equations with symmetric engine thrusts in the body-axis coordinates are

$$
\begin{aligned}
& m(\dot{u}+q w-r v)=X-m g \sin \theta+T \cos \epsilon, \\
& m(\dot{v}+r u-p w)=Y+m g \cos \theta \sin \phi, \\
& m(\dot{w}+p v-q u)=Z+m g \cos \theta \cos \phi-T \sin \epsilon, \\
& I_{x} \dot{p}+I_{x z} \dot{r}+\left(I_{z}-I_{y}\right) q r+I_{x z} q p=L, \\
& I_{y} \dot{q}+\left(I_{x}-I_{z}\right) p r+I_{x z}\left(r^{2}-p^{2}\right)=M, \\
& I_{z} \dot{r}+I_{x z} \dot{p}+\left(I_{y}-I_{x}\right) q p-I_{x z} q r=N,
\end{aligned}
$$

where

$$
\begin{aligned}
m & =\text { mass of the aircraft, } \\
{[u, v, w] } & =\text { body-axis components of the velocity of the center of mass, } \\
{[p, q, r] } & =\text { body-axis components of the angular velocity of the aircraft, } \\
{[X, Y, Z] } & =\text { body-axis aerodynamic forces about the center of mass, } \\
{[L, M, N] } & =\text { body-axis aerodynamic torques about the center of mass, } \\
(\theta, \phi) & =\text { Euler pitch and roll angles of the aircraft body axes with respect }
\end{aligned}
$$




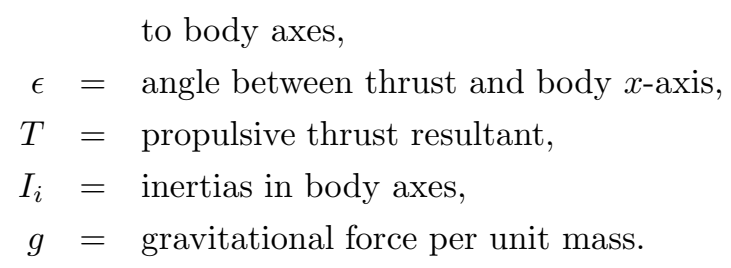

The model discussed above is under the assumption that the engine propulsive forces are symmetric (equal), so only one engine thrust term $T$ is used in these equations. In order to analyze the aircraft model with engine differentials, it is necessary to include two engine thrust terms, $T_{L}$ and $T_{R}$ which denote the left and right engine thrusts. The engine thrust differentials influence the longitudinal and lateral motions of the aircraft simultaneously, that is, these two motion components cannot be decoupled and the control design should be based on a coupled longitudinal and lateral dynamic model.

To analyze the effects of engine differentials on aircraft motion, we decompose these two engine thrusts into the $x-y$ plane, the $x-z$ plane and the $y-z$ plane which are shown in Figures 1,2 and 3 , respectively.

Figure 1 shows the vectors of the engine thrusts in the $x-y$ plane, where $\epsilon$ denotes the angle between the thrusts and the $x$-axis, $l$ represents the distance between engines and the $x-z$ plane, and $T_{L} \cos \epsilon$ and $T_{R} \cos \epsilon$ represent the engine forces along the $x$-axis.

Figure 2 shows the vectors in the $x-z$ plane, where $T_{L} \cos \epsilon$ and $T_{R} \cos \epsilon$ represent the vectors of the engine thrusts along the $x$-axis, and $T_{L} \sin \epsilon$ and $T_{R} \sin \epsilon$ represent the vectors along the $z$-axis. Here we assume the thrust vectors are aligned to pass through the $y$-axis when the engines are above or below the center of mass, so that there is no moment about the $y$-axis.

Figure 3 indicates the engine thrust components along the $z$-axis, that is, $T_{L} \sin \epsilon$ and $T_{R} \sin \epsilon$.

Unbalanced engine forces will generally produce translational motion of the center of mass and rotational motion about some axis through the center of mass. The six degree-of-freedom equations with independent engine thrusts are

$$
\begin{aligned}
& m(\dot{u}+q w-r v)=X-m g \sin \theta+\left(T_{L}+T_{R}\right) \cos \epsilon, \\
& m(\dot{v}+r u-p w)=Y+m g \cos \theta \sin \phi, \\
& m(\dot{w}+p v-q u)=Z+m g \cos \theta \cos \phi-\left(T_{L}+T_{R}\right) \sin \epsilon, \\
& I_{x} \dot{p}+I_{x z} \dot{r}+\left(I_{z}-I_{y}\right) q r+I_{x z} q p=L+l\left(T_{L}-T_{R}\right) \sin \epsilon, \\
& I_{y} \dot{q}+\left(I_{x}-I_{z}\right) p r+I_{x z}\left(r^{2}-p^{2}\right)=M, \\
& I_{z} \dot{r}+I_{x z} \dot{p}+\left(I_{y}-I_{x}\right) q p-I_{x z} q r=N+l\left(T_{L}-T_{R}\right) \cos \epsilon,
\end{aligned}
$$

where

$$
\begin{aligned}
T_{L} & =\text { left engine force } \\
T_{R} & =\text { right engine force, } \\
l & =\text { distance between engine and body } \mathrm{x} \text {-axis. }
\end{aligned}
$$

When $T_{L}$ and $T_{R}$ are equal, this model reduces to the conventional model described by (1), (2).

\section{B. Linearized Aircraft Model}

In this section, we address the linearization of the nonlinear aircraft model with engine differentials. The linearized model cannot be decoupled into separate longitudinal equations which are commonly seen in the literature.

Linearization of a nonlinear system. To linearize a nonlinear system of the form

$$
\dot{x}=f(x, U), x \in R^{n}, U \in R^{m},
$$

we first select the equilibrium values of $x_{o}, U_{o}$, that is, the values such that $\dot{x}_{o}=f\left(x_{o}, U_{o}\right)=0 .{ }^{10}$ If we expand the nonlinear equations (5) using its Taylor series about the equilibrium point $\left(x_{o}, U_{o}\right)$ and keep only the first-order terms, we obtain

$$
\dot{x} \approx f\left(x_{o}, U_{o}\right)+F\left(x-x_{o}\right)+G\left(U-U_{o}\right),
$$




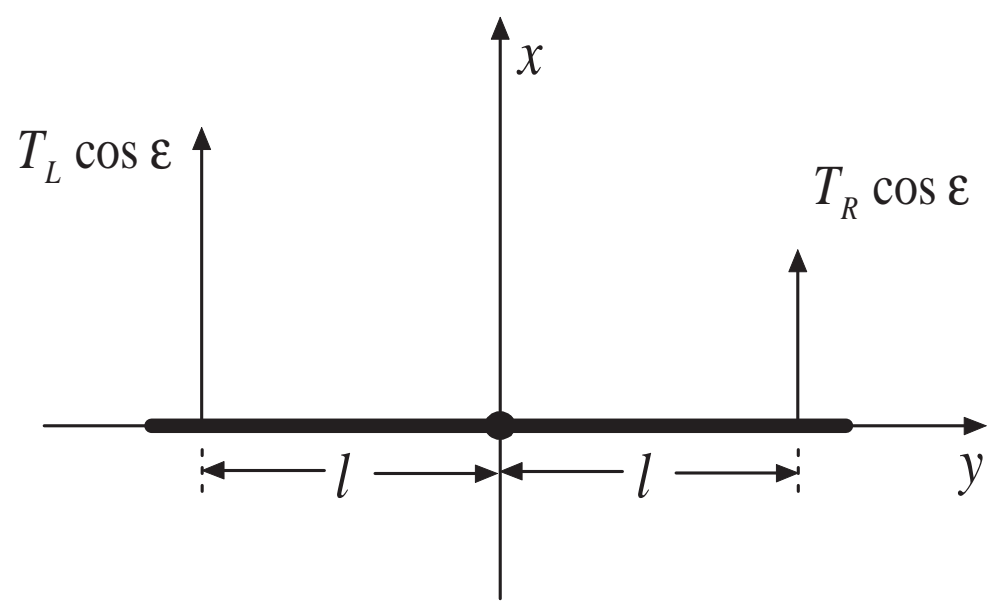

Figure 1. Engine thrust components in the $x-y$ plane.

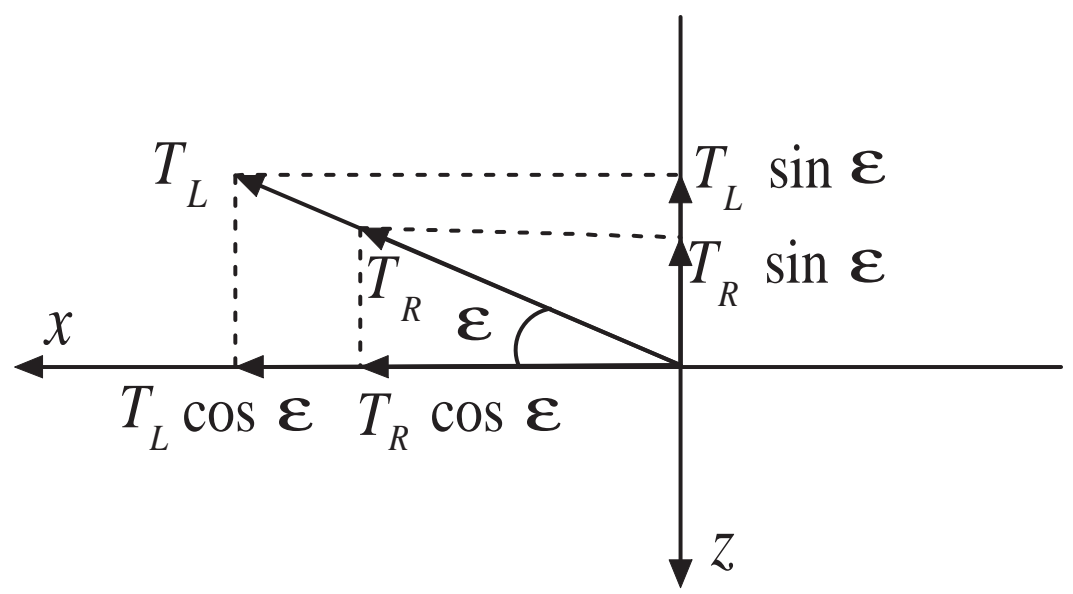

Figure 2. Engine thrust components in the $x-z$ plane.

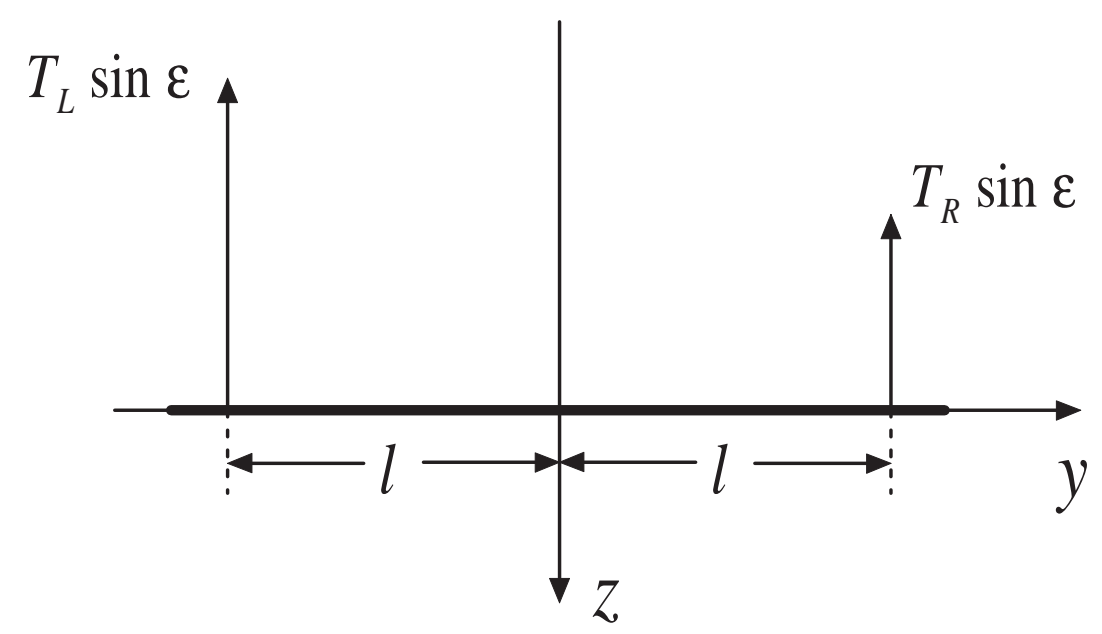

Figure 3. Engine thrust components in the $y-z$ plane. 
where

$$
F=\left[\frac{\partial f}{\partial x}\right]_{x_{o}, U_{o}}, G=\left[\frac{\partial f}{\partial U}\right]_{x_{o}, U_{o}} .
$$

Let $x=x_{o}+\delta x$ and $U=U_{o}+\delta U$, so that

$$
\dot{x}_{o}+\delta \dot{x} \approx f\left(x_{o}, U_{o}\right)+F \delta x+G \delta U .
$$

By subtracting out the equilibrium solution: $\dot{x}_{o}=f\left(x_{o}, U_{o}\right)$, we have

$$
\delta \dot{x}=F \delta x+G \delta U
$$

which is a linear differential equation approximating the motion of (5) near the equilibrium point $\left(x_{o}, U_{o}\right)$. For simplicity, the notation $\delta$ is often dropped, and $x, U$ are used to represent deviations from the equilibrium states $x_{o}$ and $U_{o}$.

Linearization of the aircraft model. Now we linearize the nonlinear aircraft model with engine thrust differentials. The state and control vectors of the linearized model are chosen as

$$
\begin{aligned}
x & =\left[\begin{array}{llllllll}
u & w & q & \theta & v & r & p & \phi
\end{array}\right]^{T} \\
U & =\left[\begin{array}{lllllll}
\delta_{e} & \delta_{t_{l}} & \delta_{t_{r}} & \delta_{a_{l}} & \delta_{a_{r}} & \delta_{r}
\end{array}\right]^{T}
\end{aligned}
$$

where, with the notation $\delta$ dropped from $\delta x$ and $\delta U$ for simplicity of presentation,

$$
\begin{aligned}
{[u, v, w] } & =\text { velocity perturbations along } \mathrm{x}, \mathrm{y}, \mathrm{z} \text { axes, } \\
{[p, q, r] } & =\text { angular velocity perturbations about } \mathrm{x}, \mathrm{y}, \mathrm{z} \text { axes, } \\
(\theta, \phi, \psi) & =\text { pitch, roll and yaw angle perturbations of the aircraft, } \\
\left(\delta_{e}, \delta_{a_{l}}, \delta_{a_{r}}, \delta_{r}\right) & =\text { elevator, left and right aileron and rudder deflections, } \\
\left(\delta_{t_{l}}, \delta_{t_{r}}\right) & =\text { left and right throttle perturbations. }
\end{aligned}
$$

In our study, we consider a steady-state rectilinear wings-level flight condition as the equilibrium point and the linearization is carried out about this point. For this steady-state flight condition, the derivatives of all states, the angular velocity components $(p, q, r)$ and the roll angle $\phi$ at the equilibrium point are all zero, that is,

$$
\begin{gathered}
{\left[\begin{array}{ccccccccc}
\dot{u} & \dot{w} & \dot{q} & \dot{\theta} & \dot{v} & \dot{r} & \dot{p} & \dot{\phi} & \dot{\psi}
\end{array}\right]_{x_{o}, U_{o}}=0} \\
p_{o}=q_{o}=r_{o}=\phi_{o}=\psi_{o}=v_{o}=0
\end{gathered}
$$

where $x_{o}$ and $U_{o}$ are determined as

$$
\begin{aligned}
x_{o} & =\left[\begin{array}{lllllllll}
u_{o} & w_{o} & 0 & \theta_{o} & 0 & 0 & 0 & 0 & 0
\end{array}\right]^{T} \\
U_{o} & =\left[\begin{array}{lllllll}
\delta_{e o} & \delta_{t_{l o}} & \delta_{t_{r o}} & \delta_{\text {alo }} & \delta_{\text {aro }} & \delta_{\text {ro }}
\end{array}\right]^{T} .
\end{aligned}
$$

To perform linearization, we derive the explicit forms of the nonlinear equations. The force equations in (3) can be written as

$$
\begin{aligned}
& \dot{u}=\frac{1}{m} X-g \sin \theta+\frac{1}{m}\left(T_{L}+T_{R}\right) \cos \epsilon-q w+r v, \\
& \dot{v}=\frac{1}{m} Y+g \cos \theta \sin \phi-r u+p w, \\
& \dot{w}=\frac{1}{m} Z+g \cos \theta \cos \phi-\frac{1}{m}\left(T_{L}+T_{R}\right) \sin \epsilon-p v+q u .
\end{aligned}
$$

The explicit form of the moment equations in (4) can be derived as

$$
\begin{aligned}
& \dot{p}=c_{1} q r+c_{2} q p+c_{3} L-c_{4} N+c_{5} l\left(T_{L}-T_{R}\right), \\
& \dot{q}=c_{6} p r+c_{7}\left(p^{2}-r^{2}\right)+c_{8} M, \\
& \dot{r}=c_{9} q p-c_{2} q r+c_{10} N-c_{11} L+c_{12} l\left(T_{L}-T_{R}\right),
\end{aligned}
$$


where

$$
\begin{array}{rlrl}
c_{1} & =\frac{I_{y} I_{z}-I_{x y}^{2}-I_{z}^{2}}{I_{x} I_{z}-I_{x z}^{2}}, & c_{2} & =\frac{I_{x z} I_{y}-I_{z} I_{x z}-I_{x} I_{x z}}{I_{x} I_{z}-I_{x z}^{2}}, \\
c_{3} & =\frac{I_{z}}{I_{x} I_{z}-I_{x z}^{2}}, & c_{4} & =\frac{I_{x z}}{I_{x} I_{z}-I_{x z}^{2}}, \\
c_{5} & =\frac{I_{z} \sin \epsilon-I_{x z} \cos \epsilon}{I_{x} I_{z}-I_{x z}^{2}}, c_{6} & =\frac{I_{z}-I_{x}}{I_{y}}, \\
c_{7} & =\frac{I_{x z}}{I_{y}}, & c_{8} & =\frac{1}{I_{y}}, \\
c_{9} & =\frac{I_{x}^{2}+I_{x z}^{2}-I_{x} I_{y}}{I_{x} I_{z}-I_{x z}^{2}}, & c_{10} & =\frac{I_{x}}{I_{x} I_{z}-I_{x z}^{2}}, \\
c_{11} & =\frac{I_{x z}}{I_{x} I_{z}-I_{x z}^{2}}, & c_{12} & =\frac{I_{x} \cos \epsilon-I_{x z} \sin \epsilon}{I_{x} I_{z}-I_{x z}^{2}} .
\end{array}
$$

There are also three kinematic equations which determine the relations between the rates of $\theta, \phi$ and $\psi$ and the body-axis components of the angular velocity $p, q$ and $r:^{7}$

$$
\begin{aligned}
\dot{\phi} & =p+\tan \theta(q \sin \phi+r \cos \phi), \\
\dot{\theta} & =q \cos \phi-r \sin \phi, \\
\dot{\psi} & =\frac{q \sin \phi+r \cos \phi}{\cos \theta} .
\end{aligned}
$$

Applying the above general linearization procedure to the aircraft equations (3), (4) and (17), we obtain the linearized aircraft model as

$$
\dot{x}=\left[\begin{array}{cc}
A_{4 \times 4}^{(1)} & A_{4 \times 5}^{(2)} \\
A_{5 \times 4}^{(3)} & A_{5 \times 5}^{(4)}
\end{array}\right] x+\left[\begin{array}{cc}
B_{4 \times 3}^{(1)} & B_{4 \times 3}^{(2)} \\
B_{5 \times 3}^{(3)} & B_{5 \times 3}^{(4)}
\end{array}\right] U,
$$

where

$$
\begin{aligned}
& A^{(1)}=\left[\begin{array}{cccc}
\bar{X}_{u} & \bar{X}_{w} & -w_{o} & -g \cos \theta_{o} \\
\bar{Z}_{u} & \bar{Z}_{w} & u_{o} & -g \sin \theta_{o} \\
\bar{M}_{u} & \bar{M}_{w} & \bar{M}_{q} & 0 \\
0 & 0 & 1 & 0
\end{array}\right], \\
& A^{(2)}=\left[\begin{array}{lllll}
0 & 0 & 0 & 0 & 0 \\
0 & 0 & 0 & 0 & 0 \\
0 & 0 & 0 & 0 & 0 \\
0 & 0 & 0 & 0 & 0
\end{array}\right] \text {, } \\
& A^{(3)}=\left[\begin{array}{cccc}
0 & 0 & 0 & 0 \\
\bar{T}_{u} & \bar{T}_{w} & 0 & 0 \\
\bar{T}_{u}^{\prime} & \bar{T}_{w}^{\prime} & 0 & 0 \\
0 & 0 & 0 & 0 \\
0 & 0 & 0 & 0
\end{array}\right] \\
& A^{(4)}=\left[\begin{array}{ccccc}
Y_{v} & -u_{o} & w_{o} & g \cos \theta_{o} & 0 \\
\bar{N}_{v} & \bar{N}_{r} & \bar{N}_{p} & 0 & 0 \\
\bar{L}_{v} & \bar{L}_{r} & \bar{L}_{p} & 0 & 0 \\
0 & \tan \theta_{o} & 1 & 0 & 0 \\
0 & \frac{1}{\cos \theta_{o}} & 0 & 0 & 0
\end{array}\right] \text {, } \\
& B^{(1)}=\left[\begin{array}{ccc}
X_{\delta_{e}} & \bar{T}_{\delta_{t_{l}}} & \bar{T}_{\delta_{t_{r}}} \\
Z_{\delta_{e}} & -\bar{T}_{\delta_{t_{l}}}^{\prime} & -\bar{T}_{\delta_{t_{r}}}^{\prime} \\
\bar{M}_{\delta_{e}} & 0 & 0 \\
0 & 0 & 0
\end{array}\right], \\
& B^{(2)}=\left[\begin{array}{lll}
0 & 0 & 0 \\
0 & 0 & 0 \\
0 & 0 & 0 \\
0 & 0 & 0
\end{array}\right]
\end{aligned}
$$




$$
\begin{aligned}
B^{(3)}= & {\left[\begin{array}{ccc}
0 & 0 & 0 \\
0 & \bar{T}_{\delta_{t_{l}}}^{\prime \prime} & -\bar{T}_{\delta_{t_{r}}}^{\prime \prime} \\
0 & \bar{T}_{\delta_{t_{l}}}^{\prime \prime \prime} & -\bar{T}_{\delta_{t_{r}}}^{\prime \prime} \\
0 & 0 & 0 \\
0 & 0 & 0
\end{array}\right], } \\
B^{(4)}= & {\left[\begin{array}{ccc}
Y_{\delta_{a l}} & Y_{\delta_{a r}} & Y_{\delta_{r}} \\
\bar{N}_{\delta_{a l}} & \bar{N}_{\delta_{a r}} & \bar{N}_{\delta_{r}} \\
\bar{L}_{\delta_{a l}} & \bar{L}_{\delta_{a r}} & \bar{L}_{\delta_{r}} \\
0 & 0 & 0 \\
0 & 0 & 0
\end{array}\right] . }
\end{aligned}
$$

In this linearized model, the non-zero terms in $A^{(3)}$ and $B^{(3)}$ represent the effect of engine thrust differentials, that is, if the left and right engine thrusts are equal to each other, those terms become zero. The other parameters in these matrices are determined as

$$
\begin{aligned}
& \bar{X}_{u}=\frac{1}{m}\left[\frac{\partial X}{\partial u}+\cos \epsilon\left(\frac{\partial T_{L}}{\partial u}+\frac{\partial T_{R}}{\partial u}\right)\right], \\
& \bar{X}_{w}=\frac{1}{m}\left[\frac{\partial X}{\partial w}+\cos \epsilon\left(\frac{\partial T_{L}}{\partial w}+\frac{\partial T_{R}}{\partial w}\right)\right], \\
& \bar{Z}_{u}=\frac{1}{m}\left[\frac{\partial Z}{\partial u}-\sin \epsilon\left(\frac{\partial T_{L}}{\partial u}+\frac{\partial T_{R}}{\partial u}\right)\right], \\
& \bar{Z}_{w}=\frac{1}{m}\left[\frac{\partial Z}{\partial w}-\sin \epsilon\left(\frac{\partial T_{L}}{\partial w}+\frac{\partial T_{R}}{\partial w}\right)\right], \\
& \bar{M}_{u}=c_{8}\left(\frac{\partial M}{\partial u}\right), \quad \bar{M}_{w}=c_{8}\left(\frac{\partial M}{\partial w}\right), \\
& \bar{M}_{q}=c_{8}\left(\frac{\partial M}{\partial q}\right), \quad \bar{T}_{u}=c_{12} l\left(\frac{\partial T_{L}}{\partial u}-\frac{\partial T_{R}}{\partial u}\right), \\
& \bar{T}_{w}=c_{12} l\left(\frac{\partial T_{L}}{\partial w}-\frac{\partial T_{R}}{\partial w}\right), \quad \bar{T}_{u}^{\prime}=c_{5} l\left(\frac{\partial T_{L}}{\partial u}-\frac{\partial T_{R}}{\partial u}\right), \\
& \bar{T}_{w}^{\prime}=c_{5} l\left(\frac{\partial T_{L}}{\partial w}-\frac{\partial T_{R}}{\partial w}\right), \quad Y_{v}=\frac{1}{m} \frac{\partial Y}{\partial v}, \\
& \bar{N}_{v}=c_{10} \frac{\partial N}{\partial v}-c_{11} \frac{\partial L}{\partial v}, \quad \bar{N}_{r}=c_{10} \frac{\partial N}{\partial r}-c_{11} \frac{\partial L}{\partial r}, \\
& \bar{N}_{p}=c_{10} \frac{\partial N}{\partial p}-c_{11} \frac{\partial L}{\partial p}, \quad \bar{L}_{v}=c_{3} \frac{\partial L}{\partial v}-c_{4} \frac{\partial N}{\partial v}, \\
& \bar{L}_{r}=c_{3} \frac{\partial L}{\partial r}-c_{4} \frac{\partial N}{\partial r}, \quad \bar{L}_{p}=c_{3} \frac{\partial L}{\partial p}-c_{4} \frac{\partial N}{\partial p}, \\
& X_{\delta_{e}}=\frac{1}{m}\left(\frac{\partial X}{\partial \delta_{e}}\right), \quad \bar{T}_{\delta_{t_{l}}}=\frac{\cos \epsilon}{m}\left(\frac{\partial T_{L}}{\partial \delta_{t_{l}}}\right), \\
& \bar{T}_{\delta_{t_{r}}}=\frac{\cos \epsilon}{m}\left(\frac{\partial T_{R}}{\partial \delta_{t_{r}}}\right), \quad Z_{\delta_{e}}=\frac{1}{m}\left(\frac{\partial Z}{\partial \delta_{e}}\right), \\
& \bar{T}_{\delta_{t_{l}}}^{\prime}=\frac{\sin \epsilon}{m}\left(\frac{\partial T_{L}}{\partial \delta_{t_{l}}}\right), \quad \bar{T}_{\delta_{t_{r}}}^{\prime}=\frac{\sin \epsilon}{m}\left(\frac{\partial T_{R}}{\partial \delta_{t_{r}}}\right), \\
& \bar{M}_{\delta_{e}}=c_{8} \frac{\partial M}{\partial \delta_{e}}, \quad \quad \bar{T}_{\delta_{t_{l}}}^{\prime \prime}=c_{12} l\left(\frac{\partial T_{L}}{\partial \delta_{t_{l}}}\right), \\
& \bar{T}_{\delta_{t_{r}}}^{\prime \prime}=c_{12} l\left(\frac{\partial T_{R}}{\partial \delta_{t_{r}}}\right), \quad \bar{T}_{\delta_{t_{l}}}^{\prime \prime \prime}=c_{5} l\left(\frac{\partial T_{L}}{\partial \delta_{t_{l}}}\right), \\
& \bar{T}_{\delta_{t_{r}}}^{\prime \prime \prime}=c_{5} l\left(\frac{\partial T_{R}}{\partial \delta_{t_{r}}}\right), \quad Y_{\delta_{a l}}=\frac{1}{m}\left(\frac{\partial Y}{\partial \delta_{a l}}\right), \\
& Y_{\delta_{a r}}=\frac{1}{m}\left(\frac{\partial Y}{\partial \delta_{a r}}\right), \quad Y_{\delta_{r}}=\frac{1}{m}\left(\frac{\partial Y}{\partial \delta_{r}}\right), \\
& \bar{N}_{\delta_{a l}}=c_{10} \frac{\partial N}{\partial \delta_{a l}}-c_{11} \frac{\partial L}{\partial \delta_{a l}}, \quad \bar{N}_{\delta_{a r}}=c_{10} \frac{\partial N}{\partial \delta_{a r}}-c_{11} \frac{\partial L}{\partial \delta_{a r}}, \\
& \bar{N}_{\delta_{r}}=c_{10} \frac{\partial N}{\partial \delta_{r}}-c_{11} \frac{\partial L}{\partial \delta_{r}}, \quad \bar{L}_{\delta_{a l}}=c_{3} \frac{\partial L}{\partial \delta_{a l}}-c_{4} \frac{\partial N}{\partial \delta_{a l}}, \\
& \bar{L}_{\delta_{a r}}=c_{3} \frac{\partial L}{\partial \delta_{a r}}-c_{4} \frac{\partial N}{\partial \delta_{a r}}, \quad \bar{L}_{\delta_{r}}=c_{3} \frac{\partial L}{\partial \delta_{r}}-c_{4} \frac{\partial N}{\partial \delta_{r}} .
\end{aligned}
$$

All $c_{i}$ parameters are defined in equation (16), and the values of the derivative signals are determined at the equilibrium point $\left(x_{o}, U_{o}\right)$ given in (14). 
This model, in which the two engine thrusts and the ailerons are taken into account separately, provides the built-in redundancy in the system to cope with some actuator failures, such as rudder failure or engine failure on one side. This feature is not captured in the conventional aircraft models that assume equal engine thrusts and aileron angles. The dynamic coupling in this aircraft model is useful for compensation of rudder or aileron failure by engine thrust differentials and introduces new challenges for flight control.

\section{Adaptive Failure Compensation}

In this section, we first formulate an actuator failure compensation problem for linear systems, and then develop an adaptive failure compensation scheme for asymptotic stabilization of the system state variables in the presence of certain actuator failures.

\section{A. Problem Formulation}

Consider the linear time-invariant system

$$
\dot{x}=A x+B u, x \in R^{n}, u \in R^{m},
$$

whose actuators $u=\left[u_{1}, u_{2}, \ldots, u_{m}\right]^{T}$ may fail during system operation, in the form: ${ }^{11}$

$$
u_{i}(t)=\bar{u}_{i}, t \geq t_{i}, i \in\{1,2, \ldots, m\},
$$

where $t_{i}$ is the unknown failure time instant and $\bar{u}_{i}$ is the unknown failure constant. An example of such actuator failures is when an aircraft control surface (such as the rudder or an aileron) is stuck at some unknown fixed position at an unknown time instant.

The control objective is to design an adaptive state feedback control signal to be applied to the actuators in $u$, to ensure closed-loop signal boundedness and asymptotic stabilization: $\lim _{t \rightarrow \infty} x(t)=0$, in the presence of unknown actuator failures which cause system parameter and structure uncertainties.

\section{B. Adaptive Compensator Design}

In the presence of actuator failures, $u(t)$ becomes

$$
u(t)=v(t)+\sigma(\bar{u}-v(t))
$$

where $v(t) \in R^{m}$ is the applied control input vector, $\bar{u}=\left[\bar{u}_{1}, \bar{u}_{2}, \ldots, \bar{u}_{m}\right]^{T}$ is the failure vector, and $\sigma$ represents the failure pattern defined as

$$
\begin{gathered}
\sigma=\operatorname{diag}\left\{\sigma_{1}, \sigma_{2}, \ldots, \sigma_{m}\right\} \\
\sigma_{i}= \begin{cases}1 & \text { if the } i \text { th actuator has failed, that is, } u_{i}=\bar{u}_{i} \\
0 & \text { otherwise. }\end{cases}
\end{gathered}
$$

The failures are assumed to occur instantaneously, i.e., $\sigma$ is a piecewise constant function of time. We denote the set of all possible failure patterns as $\bar{\Sigma}=\left\{\sigma^{i}, i=1,2, \ldots, 2^{m}\right\}$.

The system (23) can be rewritten as

$$
\dot{x}(t)=A x(t)+B(I-\sigma) v(t)+\sigma B \bar{u} .
$$

For our adaptive control design, the following assumption is needed:

Assumption III.1 $(A, B)$ is stabilizable, and $\operatorname{rank}[B(I-\sigma)]=\operatorname{rank}[B], \forall \sigma \in \Sigma$, where $\Sigma \subset \bar{\Sigma}$ is the set of recoverable failure patterns.

Next we present the adaptive scheme for the above control objective. For adaptive stabilization, similar to that in reference 12 , we choose the state feedback control law

$$
v(t)=\hat{K} x(t)+\hat{\theta}
$$


where $\hat{K}=\left[\hat{K}_{1}, \hat{K}_{2}, \ldots, \hat{K}_{m}\right]^{T} \in R^{m \times n}$ and $\hat{\theta}=\left[\hat{\theta}_{1}, \hat{\theta}_{2}, \ldots, \hat{\theta}_{m}\right]^{T} \in R^{m \times 1}$, and the adaptive law

$$
\begin{aligned}
\dot{\hat{K}}_{i} & =-\Gamma_{i} x x^{T} P b_{i}, i=1,2, \ldots, m \\
\dot{\hat{\theta}}_{i} & =-\lambda_{i} x^{T} P b_{i}, i=1,2, \ldots, m,
\end{aligned}
$$

where $\Gamma_{i}=\Gamma_{i}^{T}>0, \lambda_{i}>0, b_{i}$ is the $i$ th column of $B, i=1,2, \ldots, m$, and $P=P^{T}>0$ is the matrix satisfying the Riccati equation

$$
A^{T} P+P A-P B R^{-1} B^{T} P+Q=0 .
$$

for some chosen matrices $Q=Q^{T}>0, R=R^{T}>0$.

This adaptive actuator failure compensation scheme has the desired properties:

Theorem III.1 The control law (29), updated from (30)-(31) and applied to the system (23) subject to the actuator failures (24) under Assumption III.1, ensures that all closed-loop system signals are bounded and $\lim _{t \rightarrow \infty} x(t)=0$, for any $\sigma \in \Sigma$.

Proof: Since $(A, B)$ is stabilizable, there exists constant $K \in R^{m \times n}$ and $P \in R^{n \times n}$ such that

$$
\begin{array}{r}
P(A+B K)+(A+B K)^{T} P=-Q<0, \\
P=P^{T}>0, Q=Q^{T}>0 .
\end{array}
$$

From the condition in Assumption III.1: $\operatorname{rank}[B(I-\sigma)]=\operatorname{rank}[B], \forall \sigma \in \Sigma$, a linear combination of columns in $B$ can be expressed by a linear combination of those in $B(I-\sigma)$, that is, for each $\sigma \in \Sigma$, there exists a $K_{\sigma} \in R^{m \times n}$ such that

$$
B(I-\sigma) K_{\sigma}=B K .
$$

Therefore, for each $\sigma$, there is a $K_{\sigma}$ satisfying

$$
P\left[A+B(I-\sigma) K_{\sigma}\right]+\left[A^{T}+(I-\sigma) K_{\sigma}{ }^{T} B^{T}\right] P=-Q<0,
$$

with the same $P=P^{T}>0$ as that in (33).

Suppose that at time $t$ there are $p<m$ actuator failures in the system, that is, $u_{i}(t)=\bar{u}_{i}, \quad i=$ $i_{1}, i_{2}, \ldots, i_{p},\left\{i_{1}, i_{2}, \ldots, i_{p}\right\} \subset\{1,2, \ldots, m\}$, and that actuator failures happen at time instants $t_{k}$ (when the failure pattern $\sigma$ changes), with $t_{k}<t_{k+1}, k=1,2, \ldots, N$. For the system (28) with the adaptive controller (29)-(31), a Lyapunov function candidate can be chosen as

$$
V=\frac{1}{2} x^{T} P x+\frac{1}{2} \sum_{i \neq i_{1}, i_{2}, \ldots, i_{p}}\left(\hat{K}_{i}-K_{i}\right)^{T} \Gamma_{i}^{-1}\left(\hat{K}_{i}-K_{i}\right)+\frac{1}{2} \sum_{i \neq i_{1}, i_{2}, \ldots, i_{p}} \lambda_{i}^{-1}\left(\hat{\theta}_{i}-\theta_{i}\right)^{2},
$$

for each time interval $\left(t_{k}, t_{k+1}\right), k=0,1, \ldots, N$, with $t_{0}=0$ and $t_{N+1}=\infty$, where $K_{i}$ is the $i$ th row of $K_{\sigma}$, and $\theta_{i}$ is a solution of the following equation

$$
\sum_{i \neq i_{1}, i_{2}, \ldots, i_{p}} b_{i} \theta_{i}=-\sum_{j=i_{1}, i_{2}, \ldots, i_{p}} b_{j} \bar{u}_{j}
$$

and $\theta_{i}=0$, for $i=i_{1}, i_{2}, \ldots, i_{p}$.

The time-derivative of $V$ in each $\left(t_{k}, t_{k+1}\right)$ is

$$
\begin{aligned}
\dot{V}= & \frac{1}{2} x^{T} P(A+B(I-\sigma) \hat{K}) x+\frac{1}{2} x^{T}\left(A^{T}+\hat{K}^{T}(I-\sigma) B^{T}\right) P x+x^{T} P \sigma B \bar{u} \\
& +x^{T} P B(I-\sigma) \hat{\theta}+\sum_{i \neq i_{1}, i_{2}, \ldots, i_{p}}\left(\hat{K}_{i}-K_{i}\right)^{T} \Gamma_{i}^{-1} \dot{\hat{K}}_{i}+\sum_{i \neq i_{1}, i_{2}, \ldots, i_{p}} \lambda_{i}^{-1}\left(\hat{\theta}_{i}-\theta_{i}\right) \dot{\hat{\theta}}_{i} \\
= & \frac{1}{2} x^{T}\left[P\left(A+B(I-\sigma) K_{\sigma}\right)+\left(A^{T}+K_{\sigma}^{T}(I-\sigma) B^{T}\right) P\right] x+x^{T} P B(I-\sigma)\left(\hat{K}-\hat{K}_{\sigma}\right) x \\
& +\sum_{i=j_{1}, i_{2}, \ldots, i_{p}} x^{T} P b_{j} \bar{u}_{j}+\sum_{i \neq i_{1}, i_{2}, \ldots, i_{p}} x^{T} P b_{i} \theta_{i}+x^{T} P B(I-\sigma)(\hat{\theta}-\theta) \\
& +\sum_{i \neq i_{1}, i_{2}, \ldots, i_{p}}\left(\hat{K}_{i}-K_{i}\right)^{T} \Gamma_{i}^{-1} \dot{\hat{K}}_{i}+\sum_{i \neq i_{1}, i_{2}, \ldots, i_{p}} \lambda_{i}^{-1}\left(\hat{\theta}_{i}-\theta_{i}\right) \dot{\hat{\theta}}_{i} \\
= & -\frac{1}{2} x^{T} Q x \leq 0 .
\end{aligned}
$$


It follows that $x \in L^{2} \cap L^{\infty}$, and $\hat{K}_{i} \in L^{\infty}$ and $\hat{\theta}_{i} \in L^{\infty}$ for $i \neq i_{1}, i_{2}, \ldots, i_{p}$. From (30), we have

$$
\left[\Gamma_{1}^{-1} \dot{\hat{K}}_{1}, \Gamma_{2}^{-1} \dot{\hat{K}}_{2}, \ldots, \Gamma_{m}^{-1} \dot{\hat{K}}_{m}\right]=-x x^{T} P B \text {. }
$$

Since $B$ can be represented by a linear combination of $b_{i}, i \neq i_{1}, i_{2}, \ldots, i_{p}, \Gamma_{i}^{-1} \dot{\hat{K}}_{i}, i \in\left\{i_{1}, i_{2}, \ldots, i_{p}\right\}$, is also a linear combination of $\Gamma_{i}^{-1} \dot{\hat{K}}_{i}, i \neq i_{1}, i_{2}, \ldots, i_{p}$. This implies that $\hat{K}_{i} \in L^{\infty}$ for $i=i_{1}, i_{2}, \ldots, i_{p}$, and similarly, that $\hat{\theta}_{i} \in L^{\infty}$ for $i=i_{1}, i_{2}, \ldots, i_{p}$.

The function $V$ is not continuous at $t_{k}, k=0,1, \ldots, N$, and only has finite value jumps at those time instants. So we can obtain that $x \in L^{2} \cap L^{\infty}, \hat{K} \in L^{\infty}$, and $\hat{\theta} \in L^{\infty}, \forall t \geq 0$. Because $v(t) \in L^{\infty}$ and $\dot{x} \in L^{\infty}$, given that $x(t) \in L^{2}$, we also have $\lim _{t \rightarrow \infty} x(t)=0$.

This adaptive failure compensation scheme is applicable to the linearized aircraft dynamic model around an equilibrium point $\left(x_{o}, U_{o}\right)$ which represents the steady-state rectilinear wing-level flight condition. The physical meaning of a non-zero state vector $x(t)$ in $(23)$ is that the state of the nonlinear system is perturbed away from the equilibrium point, i.e., the steady-state condition. The adaptive scheme is applicable when the actuator failure patterns belong to a set specified by Assumption III.1 and some of the $m$ actuators in $u=\left[u_{1}, u_{2}, \ldots, u_{m}\right]^{T}$ may fail. For example, for the rudder failure case, $u_{6}=\delta_{r}=\bar{u}_{6}$ may happen for an unknown value $\bar{u}_{6}$ which is an unknown value not influenced by the commaneded control input signal.

The result of Theorem III.1 implies that under Assumption III.1 the adaptive control scheme is able to achieve asymptotic regulation of $x(t)$ to zero. This means that, under the condition of Assumption III.1 (which is indeed satisfied in the case of rudder failure), the aircraft will be automatically returned to the steady-state flight condition under both normal and upset conditions, (the condition is unknown to the feedback controller). This will be demonstrated in Section IV in detail.

\section{Application to Aircraft Flight Control: Regulation}

In this section, we present simulation results to show the effectiveness of our adaptive scheme, applied to compensation of aircraft rudder and aileron failures, primarily using engine thrust differentials. We will first present an aircraft model and the adaptive design for this model, then introduce a nominal control design, and finally give the simulation results of adaptive actuator failure compensation.

\section{A. Aircraft Flight Control System}

For our simulation study, we use a large transport aircraft model (similar to Boeing 747). The airplane flies at a velocity of $774 \mathrm{ft} / \mathrm{sec}$ and an altitude of $40 \mathrm{kft}$. The linearized dynamic model is

$$
\dot{x}(t)=\left[\begin{array}{ll}
A_{4 \times 4}^{(1)} & A_{4 \times 5}^{(2)} \\
A_{5 \times 4}^{(3)} & A_{5 \times 5}^{(4)}
\end{array}\right] x(t)+\left[\begin{array}{ll}
B_{4 \times 3}^{(1)} & B_{4 \times 3}^{(2)} \\
B_{5 \times 3}^{(3)} & B_{5 \times 3}^{(4)}
\end{array}\right] U(t),
$$

where

$$
\begin{aligned}
& x=\left[\begin{array}{lllllllll}
u & w & q & \theta & v & r & p & \phi & \psi
\end{array}\right]^{T}, \\
& U=\left[\begin{array}{llllll}
\delta_{e} & \delta_{t_{l}} & \delta_{t_{r}} & \delta_{a_{l}} & \delta_{a_{r}} & \delta_{r}
\end{array}\right]^{T}, \\
& A^{(1)}=\left[\begin{array}{cccc}
-0.003 & 0.039 & 0 & -0.322 \\
-0.065 & -0.319 & 7.74 & 0 \\
0.020 & -0.101 & -0.429 & 0 \\
0 & 0 & 1 & 0
\end{array}\right] \text {, } \\
& A^{(2)}=\left[\begin{array}{lllll}
0 & 0 & 0 & 0 & 0 \\
0 & 0 & 0 & 0 & 0 \\
0 & 0 & 0 & 0 & 0 \\
0 & 0 & 0 & 0 & 0
\end{array}\right] \\
& A^{(3)}=\left[\begin{array}{cccc}
0 & 0 & 0 & 0 \\
0.001 & 0.001 & 0 & 0 \\
-0.001 & -0.001 & 0 & 0 \\
0 & 0 & 0 & 0 \\
0 & 0 & 0 & 0
\end{array}\right]
\end{aligned}
$$

10 of 25 


$$
\begin{aligned}
& A^{(4)}=\left[\begin{array}{ccccc}
-0.0558 & -7.74 & 0 & 0.322 & 0 \\
0.0773 & -0.115 & -0.0318 & 0 & 0 \\
-0.394 & 0.388 & -0.465 & 0 & 0 \\
0 & 0 & 1 & 0 & 0 \\
0 & 1 & 0 & 0 & 0
\end{array}\right] \text {, } \\
& B^{(1)}=\left[\begin{array}{ccc}
0.01 & 1 & 1 \\
-0.18 & -0.04 & -0.04 \\
-1.16 & 0.598 & 0.598 \\
0 & 0 & 0
\end{array}\right] \text {, } \\
& B^{(2)}=\left[\begin{array}{lll}
0 & 0 & 0 \\
0 & 0 & 0 \\
0 & 0 & 0 \\
0 & 0 & 0
\end{array}\right] \text {, } \\
& B^{(3)}=\left[\begin{array}{ccc}
0 & 0 & 0 \\
0 & 0.8 & -0.7 \\
0 & -0.5 & 0.6 \\
0 & 0 & 0 \\
0 & 0 & 0
\end{array}\right] \\
& B^{(4)}=\left[\begin{array}{ccc}
0.03 & -0.03 & 0.0564 \\
0.0036 & 0.0036 & -0.4750 \\
0.0715 & 0.0715 & 0.153 \\
0 & 0 & 0 \\
0 & 0 & 0
\end{array}\right] \text {. }
\end{aligned}
$$

The non-zero terms in $A^{(3)}$ and $B^{(3)}$ represent the engine thrust differential effect. In this model, the units of the variables are $\mathrm{ft}$, sec and crad (centi-radians).

In this study, we consider the rudder and aileron failures compensation problem: during the system operation, the rudder or an aileron is stuck at an unknown constant value at an unknown time instant, that is, both are unknown to the feedback controller.

According to the adaptive control algorithm in Section B, the feedback control law is

$$
v_{d}(t)=\hat{K} x(t)+\hat{\theta},
$$

with $\hat{K}=\left[\hat{K}_{1}, \hat{K}_{2}, \ldots, \hat{K}_{6}\right]^{T} \in R^{6 \times 9}$ and $\hat{\theta}=\left[\hat{\theta}_{1}, \hat{\theta}_{2}, \ldots, \hat{\theta}_{6}\right]^{T} \in R^{6 \times 1}$ updated by

$$
\begin{aligned}
\dot{\hat{K}}_{i} & =-\Gamma_{i} x x^{T} P b_{i}, i=1,2, \ldots, 6 \\
\dot{\hat{\theta}}_{i} & =-\lambda_{i} x^{T} P b_{i}, i=1,2, \ldots, 6,
\end{aligned}
$$

where $\Gamma_{i}=\Gamma_{i}^{T}>0, \lambda_{i}>0, b_{i}$ is the $i$ th column of $B, i=1,2, \ldots, 6$, and $P=P^{T}>0$ is such that

$$
A^{T} P+P A-P B R^{-1} B^{T} P+Q=0 .
$$

In our simulation, we choose the initial value of the state vector as

$$
x_{0}=\left[\begin{array}{lllllllll}
2 & -8 & -0.3 & 4 & 0.7 & -0.3 & 1 & -3 & 3
\end{array}\right]^{T} .
$$

This initial state vector represents a non-steady state (perturbed) flight condition in which the aircraft is climbing with a $2 \mathrm{ft} / \mathrm{sec}$ velocity perturbation on $x$-axis and $8 \mathrm{ft} / \mathrm{sec}$ velocity perturbation on $z$-axis and 0.7 $\mathrm{ft} / \mathrm{sec}$ on $y$-axis. The yaw rate and pitch rate are $-0.3 \mathrm{crad} / \mathrm{sec}(-0.17$ degree $/ \mathrm{sec})$. The pitch angle is $4 \mathrm{crads}$ (2.29 degrees) and roll angle are -3 crads (-1.72 degrees). The roll rate is $1 \mathrm{crad} / \mathrm{sec}(0.57 \mathrm{deg} / \mathrm{sec})$ and the yaw angle is 3 crads (1.72 degrees).

For controller design, $Q$ is chosen as $\operatorname{diag}\{1,1,1,1,7,1,1,6,5\}$ and $R$ is chosen as $5 I_{6}$ to obtain satisfactory convergence of the state vector in simulation study.

We consider two types of constant rudder failures in this simulation study. The first type is defined as

$$
U_{6}(t)=U_{6}\left(t_{f}\right), t \geq t_{f},
$$

11 of 25 
where $t_{f}$ is the failure instant. This type implies that the rudder is stuck at the position of the time when the failure happens. The second type of failure we consider is defined as

$$
U_{6}(t)=2 \mathrm{crad}, t \geq t_{f},
$$

where $t_{f}$ is the failure instant.

The aileron failure we will consider is

$$
U_{5}(t)=0 \mathrm{crad}, t \geq t_{f},
$$

which means that the right aileron will be stuck at zero after $t_{f}$.

The control signal $v_{d}(t)$ is applied to the plant (40) subject to the actuator failure (47), (48), or (49).

In the following simulation study, we will examine eight different cases:

(I) System responses with nominal controller for rudder failure (47),

(II) System responses with nominal controller for rudder failure (48),

(III) System responses with adaptive failure compensation for rudder failure (47),

(IV) System responses with adaptive failure compensation for rudder failure (48),

(V) System responses with adaptive compensation for rudder failure $(48)(x(0)=0)$,

(VI) System responses with adaptive compensation switched on after failure (47),

(VII) System responses with adaptive compensation switched on after failure (48), and

(VIII)System responses with adaptive failure compensation for aileron failure (49);

where Case (I) and (II) are for the nominal design which we will present in the next subsection, while Case (III)-(VIII) examine the effectiveness of the adaptive failure compensation scheme. In particular, the Case (VI) and (VII) are presented as comparisons with Case (I) and (II).

\section{B. System Performance with A Nominal Design}

In this subsection, we present a nominal control design based on a linear quadratic regulator (LQR). ${ }^{13}$ The feedback control law is

$$
v_{d}(t)=K x(t)
$$

where $K \in R^{6 \times 9}$ is a feedback gain matrix chosen to minimize a quadratic performance index

$$
J=\int_{0}^{\infty}\left(x^{T} Q x+u^{T} R u\right) d t
$$

where $Q=Q^{T}>0$ and $R=R^{T}>0$ are the weighting matrices. For $P=P^{T}>0$ satisfying

$$
A^{T} P+P A-P B R^{-1} B^{T} P+Q=0,
$$

the feedback gain $K$ is given as

$$
K=-R^{-1} B^{T} P .
$$

This LQR design ensures asymptotic stability of the closed-loop system. However, in presence of unknown rudder failures, the nominal performance is no longer satisfactory. Typical responses are shown in the simulation results in Case (I) and Case (II), which indicate that some of the states cannot be asymptotically stabilized.

Case (I). As described in Section A, Case (I) shows the system performance with nominal controller design in the presence of rudder failure (47). The failure instant is $t_{f}=10$ seconds and the initial values for the states are described in (46). The results are shown in Figures 4 and 5.

From the figures we can see that some states cannot be stabilized with this nominal controller, such as yaw angle $\psi$.

Case (II). In this case, we show another situation of the system performance with the nominal controller in the presence of rudder failure (48). The failure instant is $t_{f}=30$ seconds. Other parameters for simulation are the same with the previous case. The results are shown in Figures 6 and 7.

In this case, some states, such as yaw angle $\psi$ and $x$-axis velocity $u$, cannot be stabilized, which is similar with Case (I). The results in Case (I) and Case (II) show that the LQR design is not capable of coping with the unknown rudder failure and cannot achieve satisfactory performance.

\section{2 of 25}



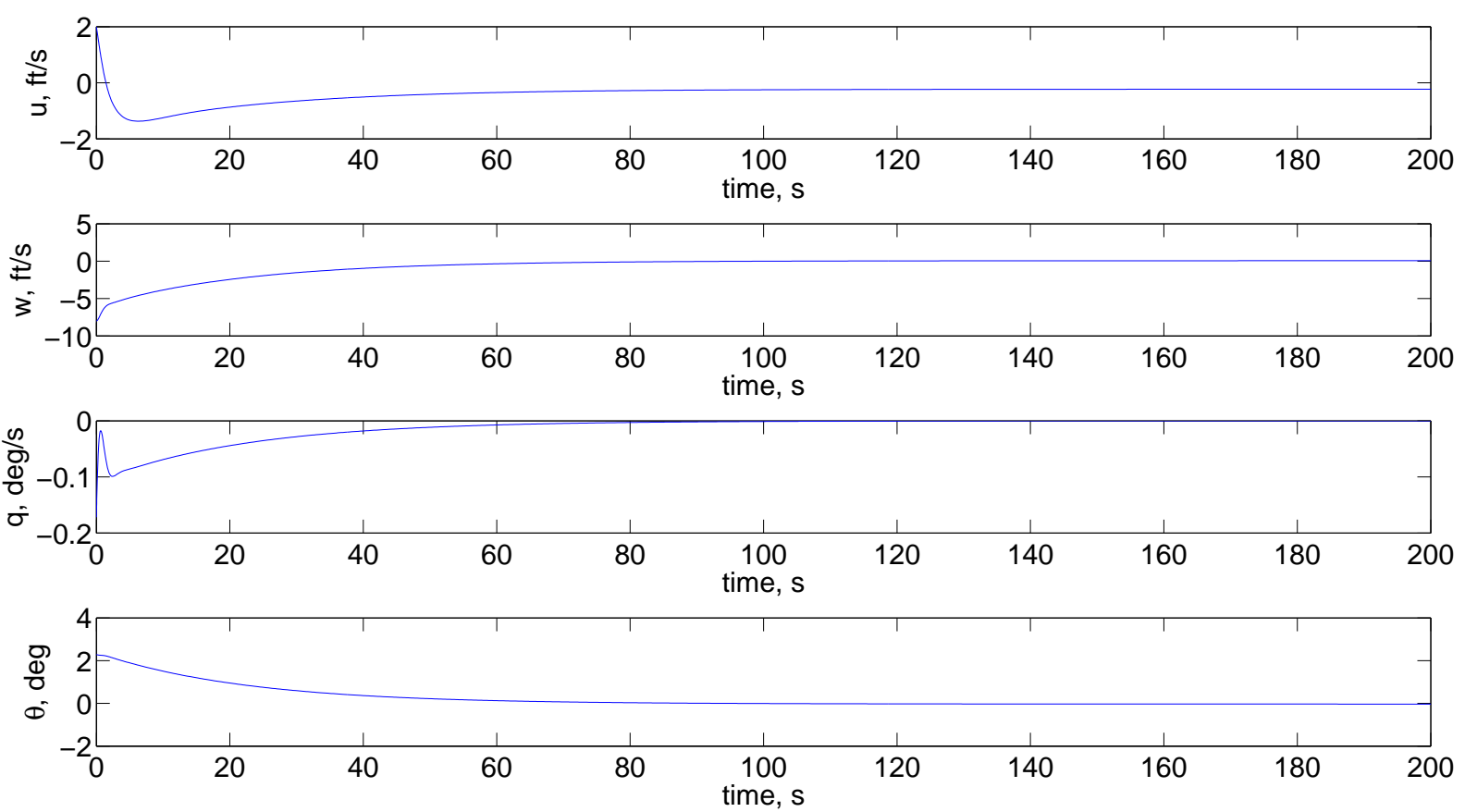

Figure 4. System states $x_{1}=u, x_{2}=w, x_{3}=q, x_{4}=\theta$ (Case I).
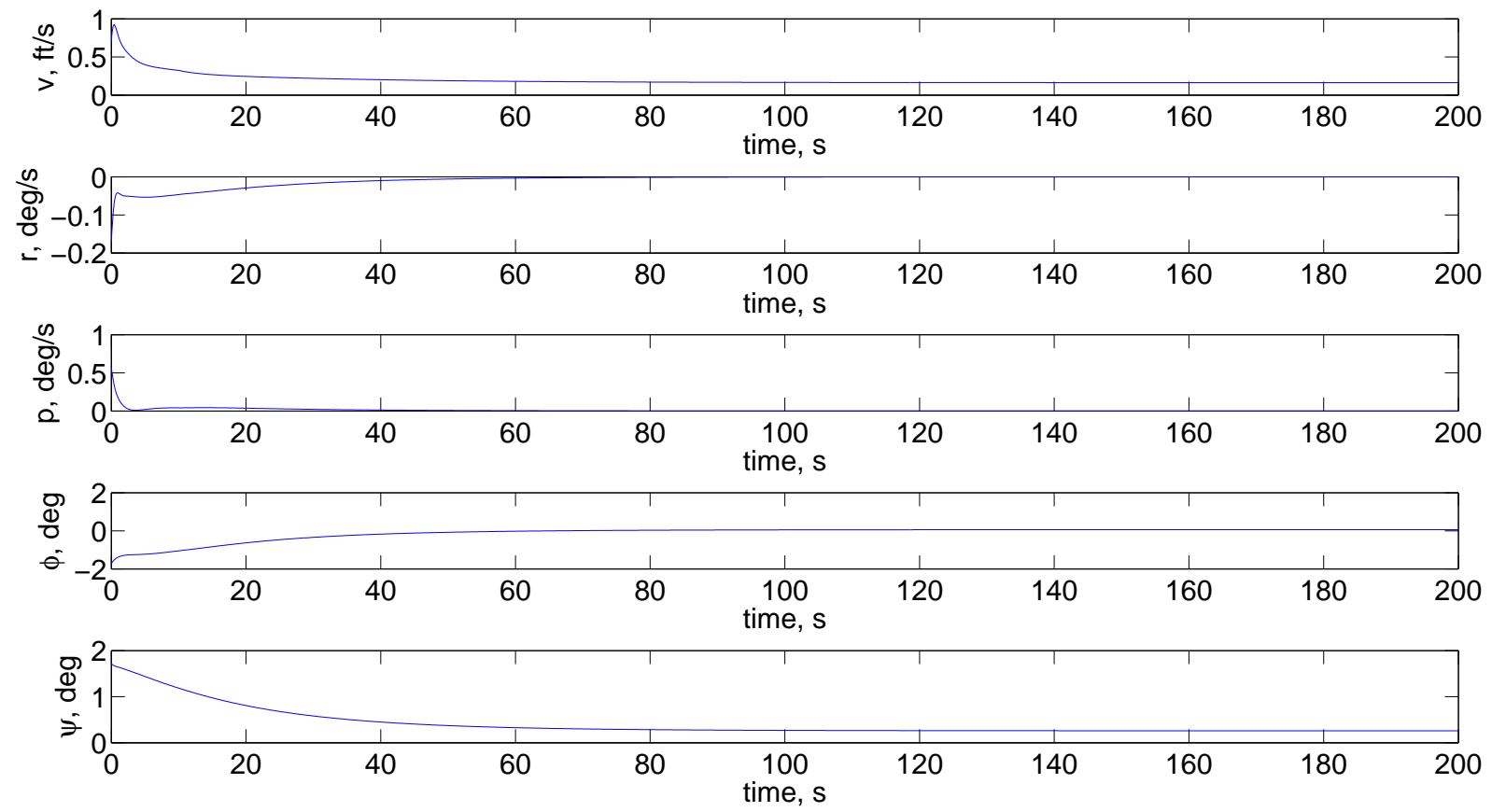

Figure 5. System states $x_{5}=v, x_{6}=r, x_{7}=p, x_{8}=\phi, x_{9}=\psi$ (Case I). 

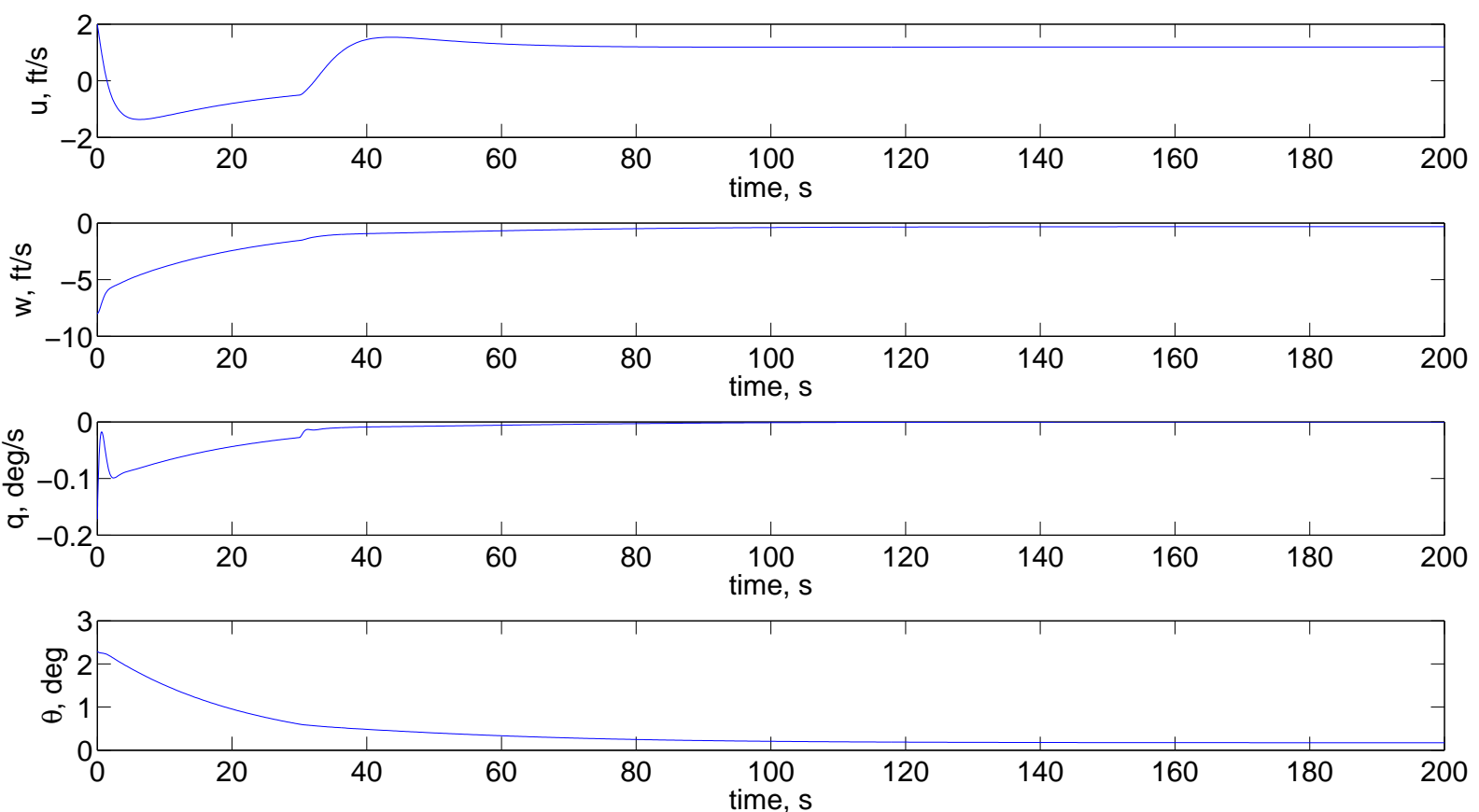

Figure 6. System states $x_{1}=u, x_{2}=w, x_{3}=q, x_{4}=\theta$ (Case II).
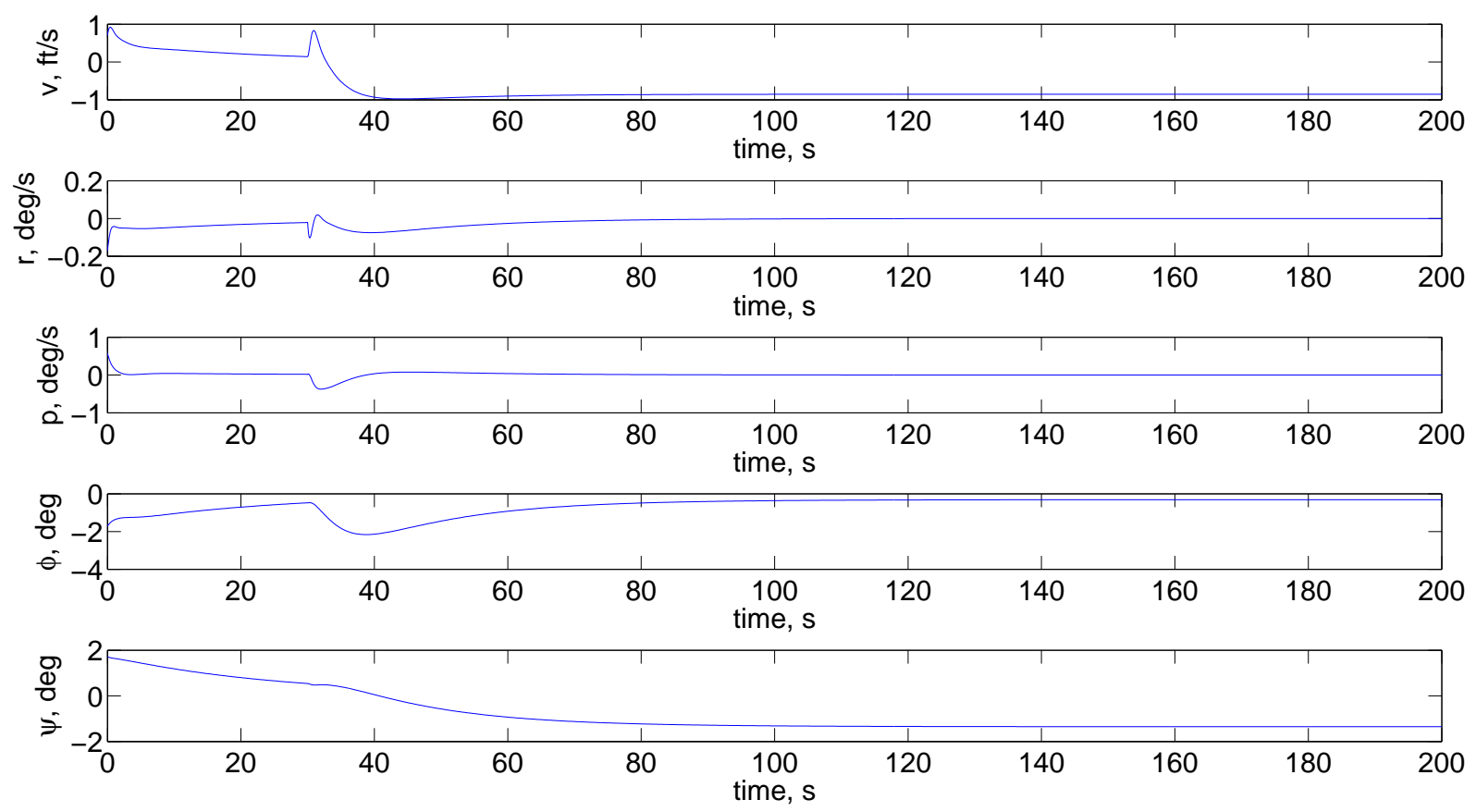

Figure 7. System states $x_{5}=v, x_{6}=r, x_{7}=p, x_{8}=\phi, x_{9}=\psi$ (Case II). 


\section{System Performance with Adaptive Failure Compensation}

In this subsection, we present the simulation results for the control system with the adaptive actuator failure compensation control design given in Section A, to show the performance improvement by adaptive failure compensation of an uncertain rudder failure.

Case (III). In this case, we consider the rudder failure (47). The failure instant is chosen as $t_{f}=$ 10 seconds. The initial values for the states are defined in (46). The elements in $\Gamma_{i}(i=1,2, \ldots, 6)$ are chosen as $\left[\begin{array}{lllllllll}0.01 & 0.01 & 0.01 & 0.01 & 0.08 & 0.02 & 0.02 & 4 & 4\end{array}\right]$, and $\lambda_{i}(i=1,2, \ldots, 6)$ are chosen as $\left[\begin{array}{llllll}0.02 & 0.4 & 0.4 & 0.4 & 0.4 & 0.03\end{array}\right]$. The system response and some control inputs are shown in Figures 8, 9 and 10. We can see from the results that all the states can be stabilized with adaptive failure compensation in the presence of rudder failure.
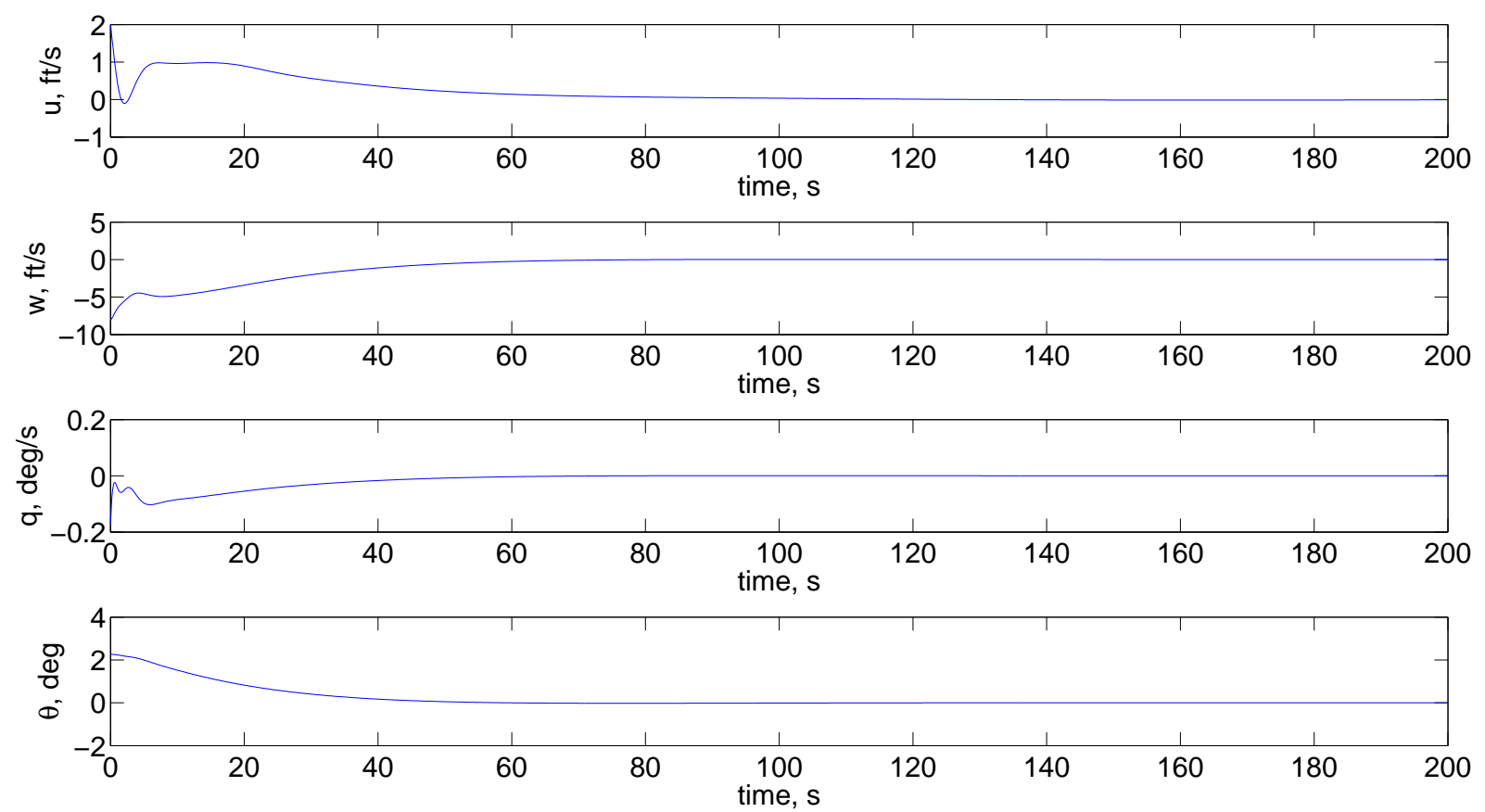

Figure 8. System states $x_{1}=u, x_{2}=w, x_{3}=q, x_{4}=\theta$ (Case III).

Case (IV). In this case, the parameter setting is the same with the last case except that the rudder failure (48) is consider in this case. The failure instant is $t_{f}=30$ second. The results are shown in Figures 11,12 and 13.

Case $(\mathbf{V})$. In Case $(\mathrm{V})$, we set the initial values of the states to be zero, i.e., the airplane is in steady wings-level flight. Without the transient response at the beginning, the influence of the rudder failure on the states is accentuated. The rudder failure (48) is considered with failure instant $t_{f}=30$ seconds. The elements in $\Gamma_{i}(i=1,2, \ldots, 6)$ are chosen as $\left[\begin{array}{lllllllll}1 & 1 & 1 & 1 & 1 & 1 & 1 & 4 & 9\end{array}\right]$, and $\lambda_{i}(i=1,2, \ldots, 6)$ are chosen as [ $\left[\begin{array}{llllll}1 & 1 & 1 & 3 & 3 & 2\end{array}\right]$. The results are shown in Figures 14, 15 and 16.

Case (VI). In Case (VI) and (VII), we will show the simulation results of system performance with adaptive failure compensation scheme switched on sometime after the failure happens. In Case (VI), we consider the failure (47) with failure instant $t_{f}=10$ seconds. And at $t=60$ seconds the adaptive scheme is switched on. The elements in $\Gamma_{i}(i=1,2, \ldots, 6)$ are chosen as $\left[\begin{array}{lllllllll}0.01 & 0.01 & 0.01 & 0.01 & 0.08 & 0.02 & 0.02 & 4 & 4\end{array}\right]$, and $\lambda_{i}(i=1,2, \ldots, 6)$ are chosen as $\left[\begin{array}{llllll}0.02 & 0.4 & 0.4 & 0.4 & 0.4 & 0.03\end{array}\right]$. The results are shown in Figures 17 , 18 and 19.

Case (VII). In Case (VII), the parameters for simulation are the same with Case (VI) except that the failure (48) is considered with failure instant $t_{f}=30$ seconds. The adaptive compensation scheme is switched on after $t=60$ seconds. The results are shown in Figures 20, 21 and 22. From the simulation results in 

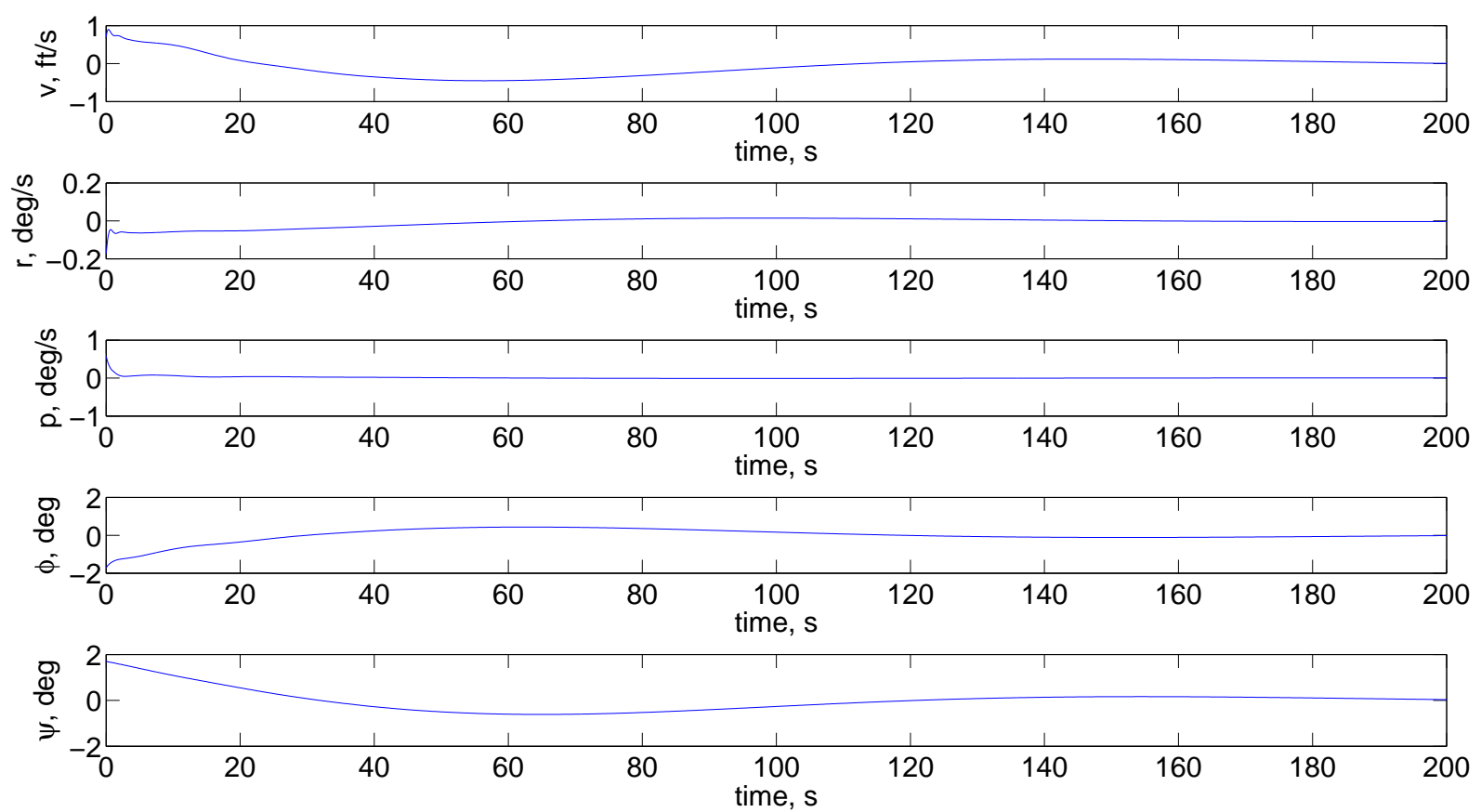

Figure 9. System states $x_{5}=v, x_{6}=r, x_{7}=p, x_{8}=\phi, x_{9}=\psi$ (Case III).
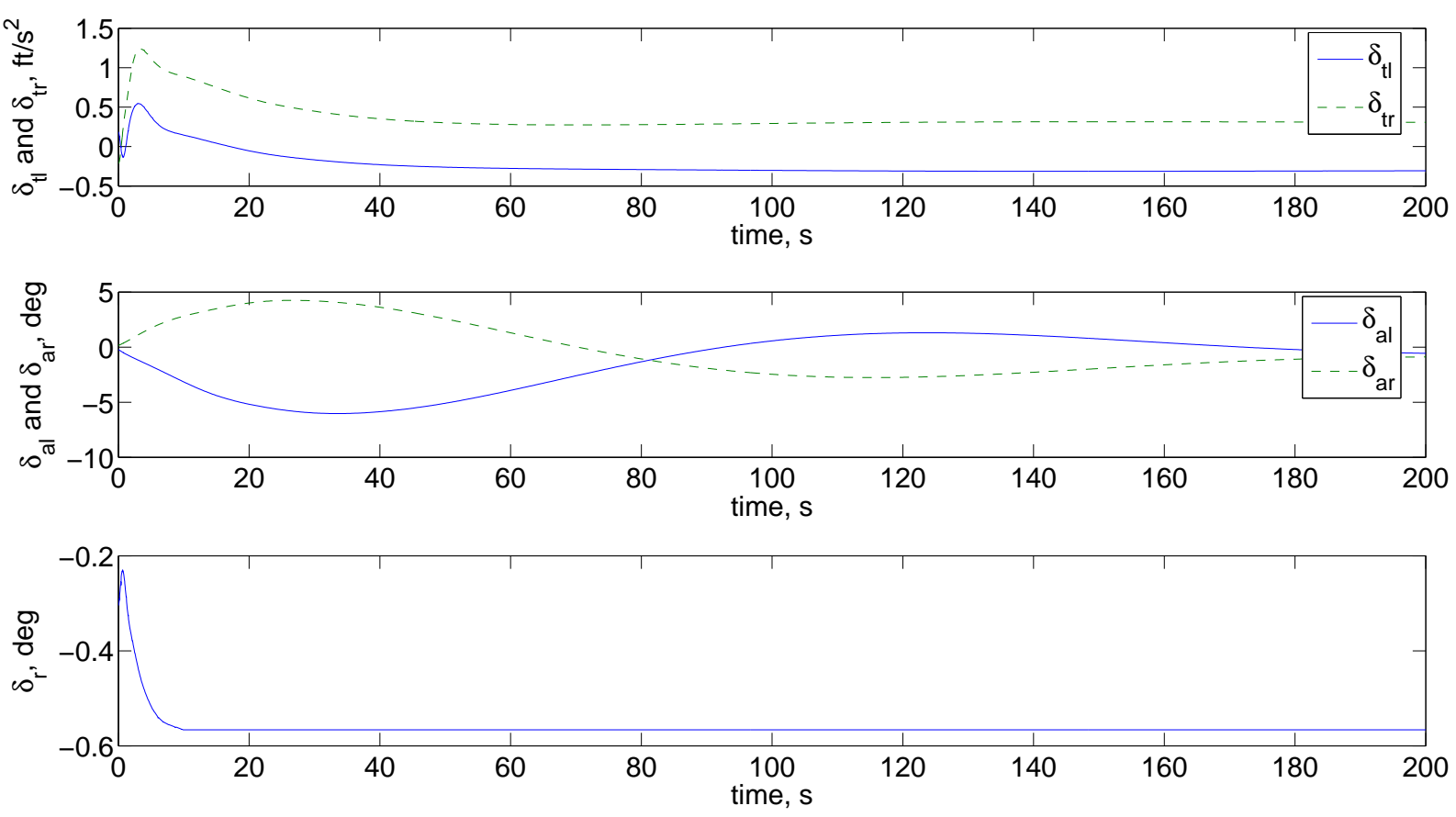

Figure 10. Control signals: $\delta_{t l}, \delta_{t r}, \delta_{a l}, \delta_{a r}$ and $\delta_{r}$ (Case III). 

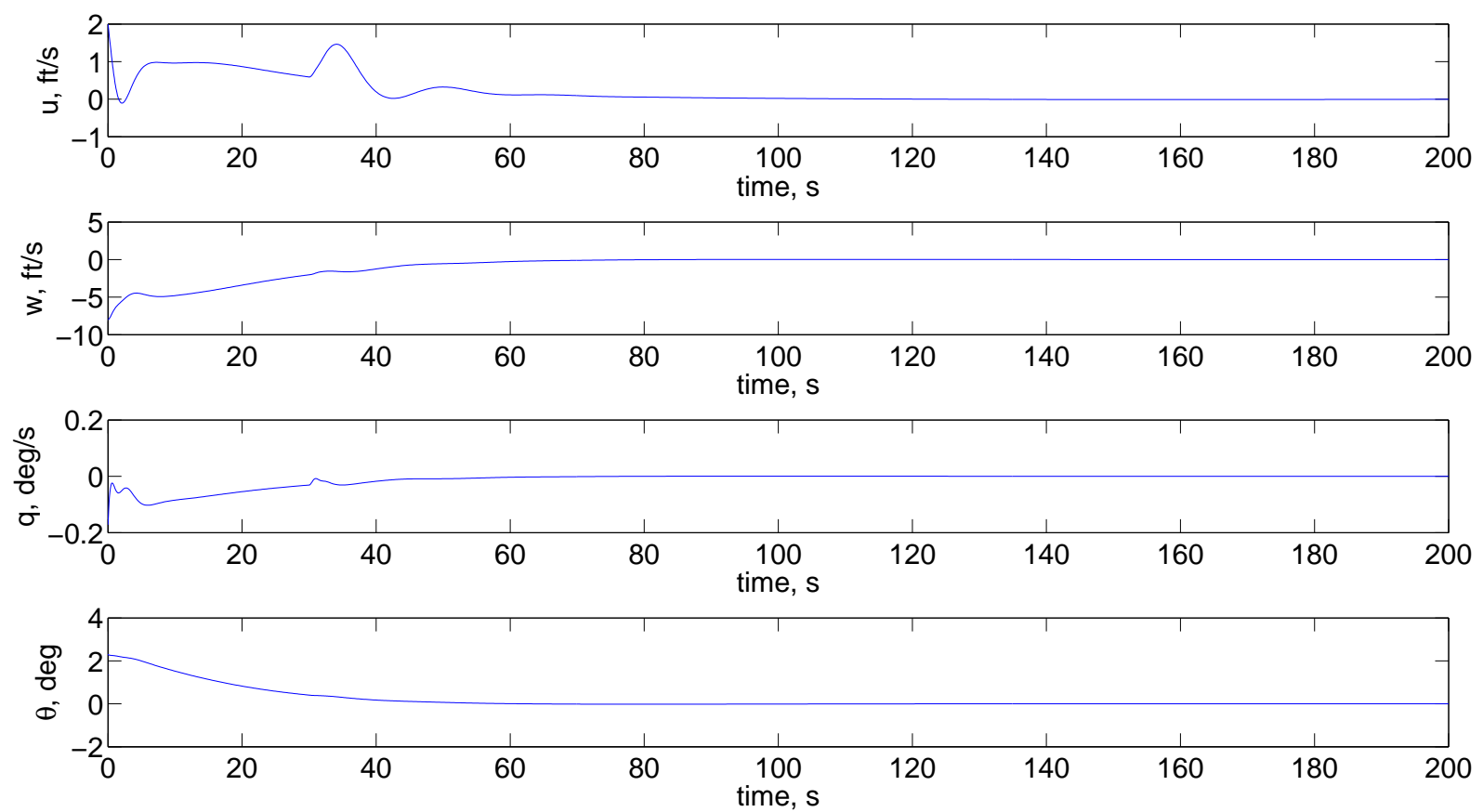

Figure 11. System states $x_{1}=u, x_{2}=w, x_{3}=q, x_{4}=\theta$ (Case IV).
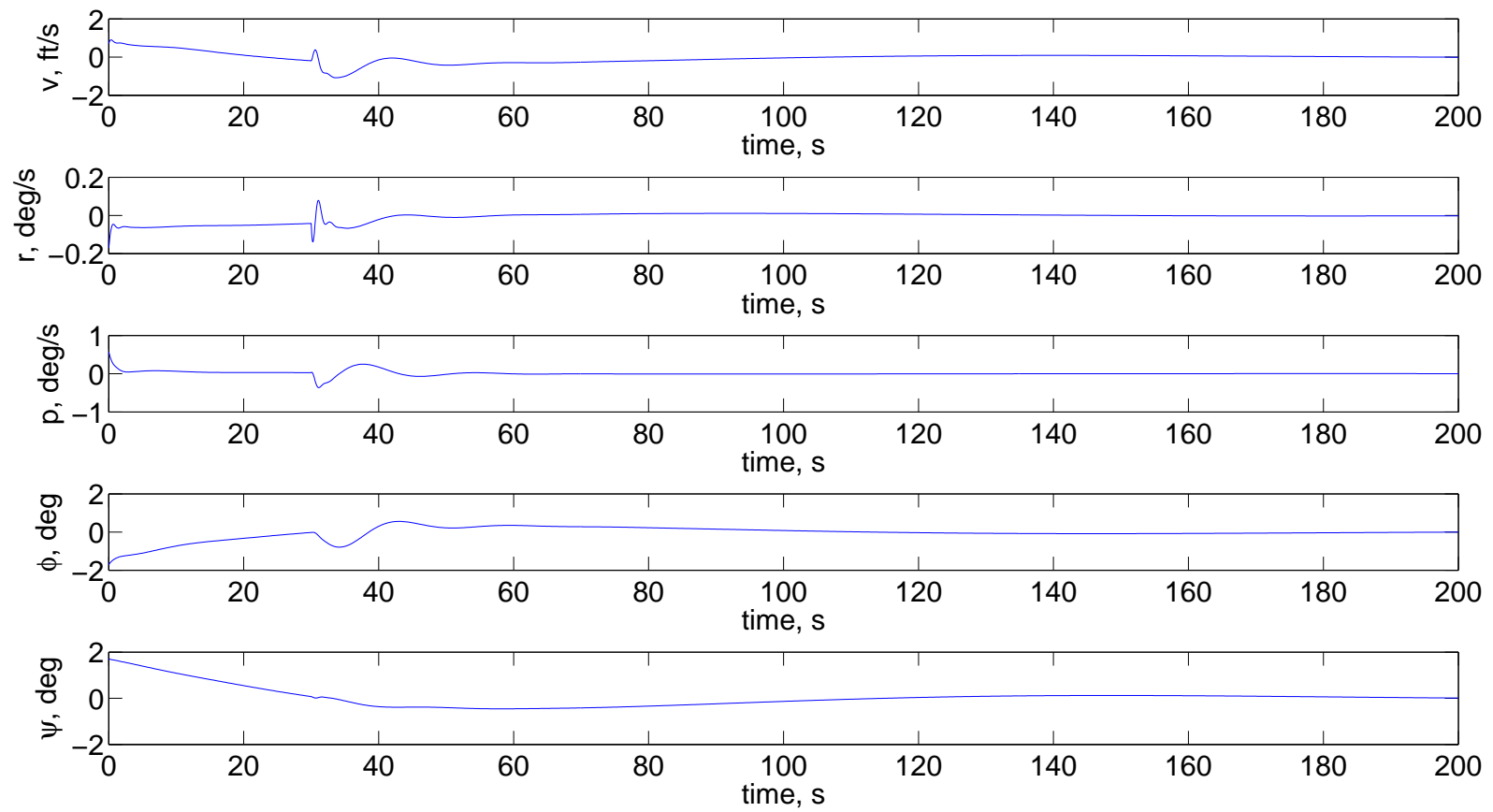

Figure 12. System states $x_{5}=v, x_{6}=r, x_{7}=p, x_{8}=\phi, x_{9}=\psi$ (Case IV). 

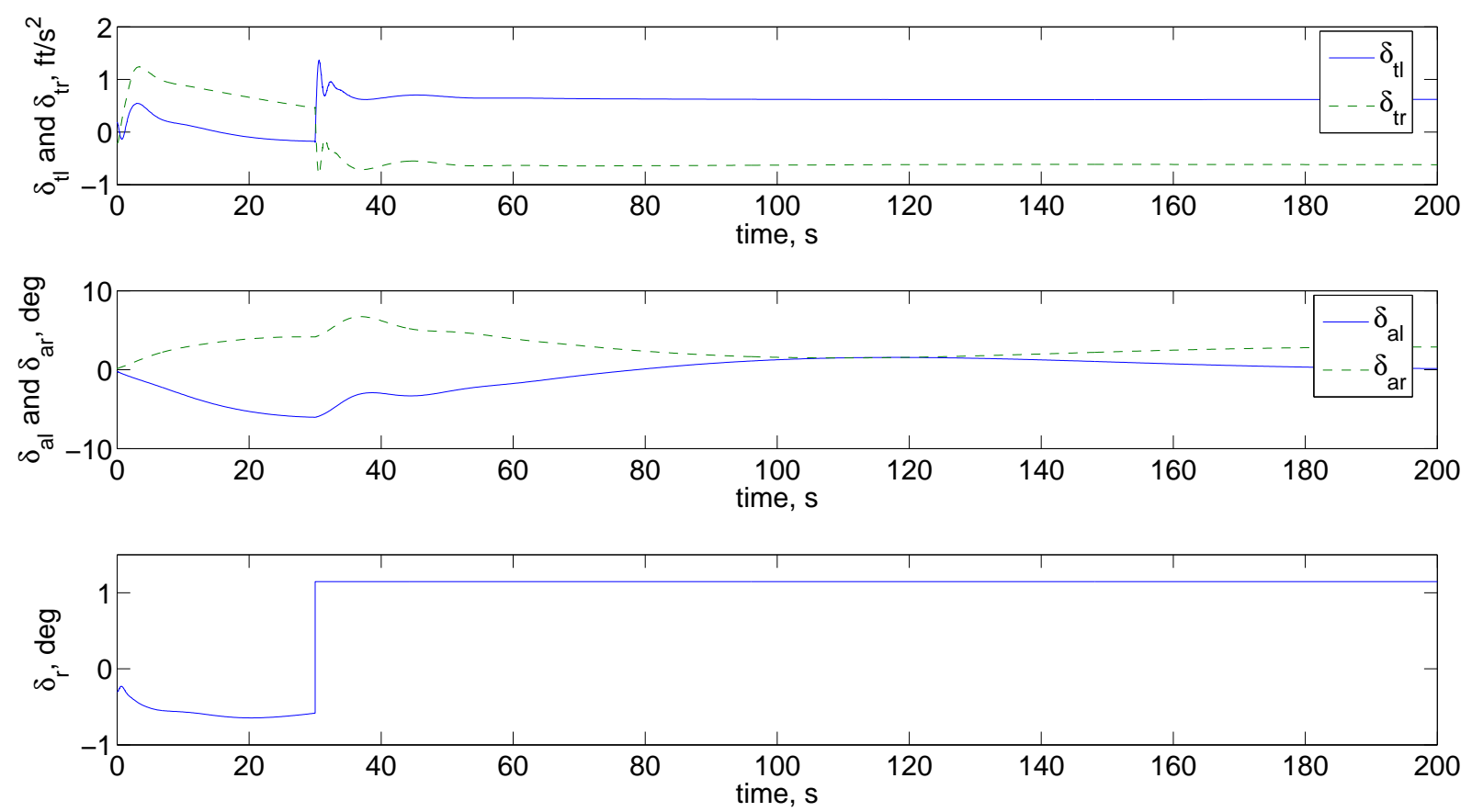

Figure 13. Control signals: $\delta_{t l}, \delta_{t r}, \delta_{a l}, \delta_{a r}$ and $\delta_{r}$ (Case IV).
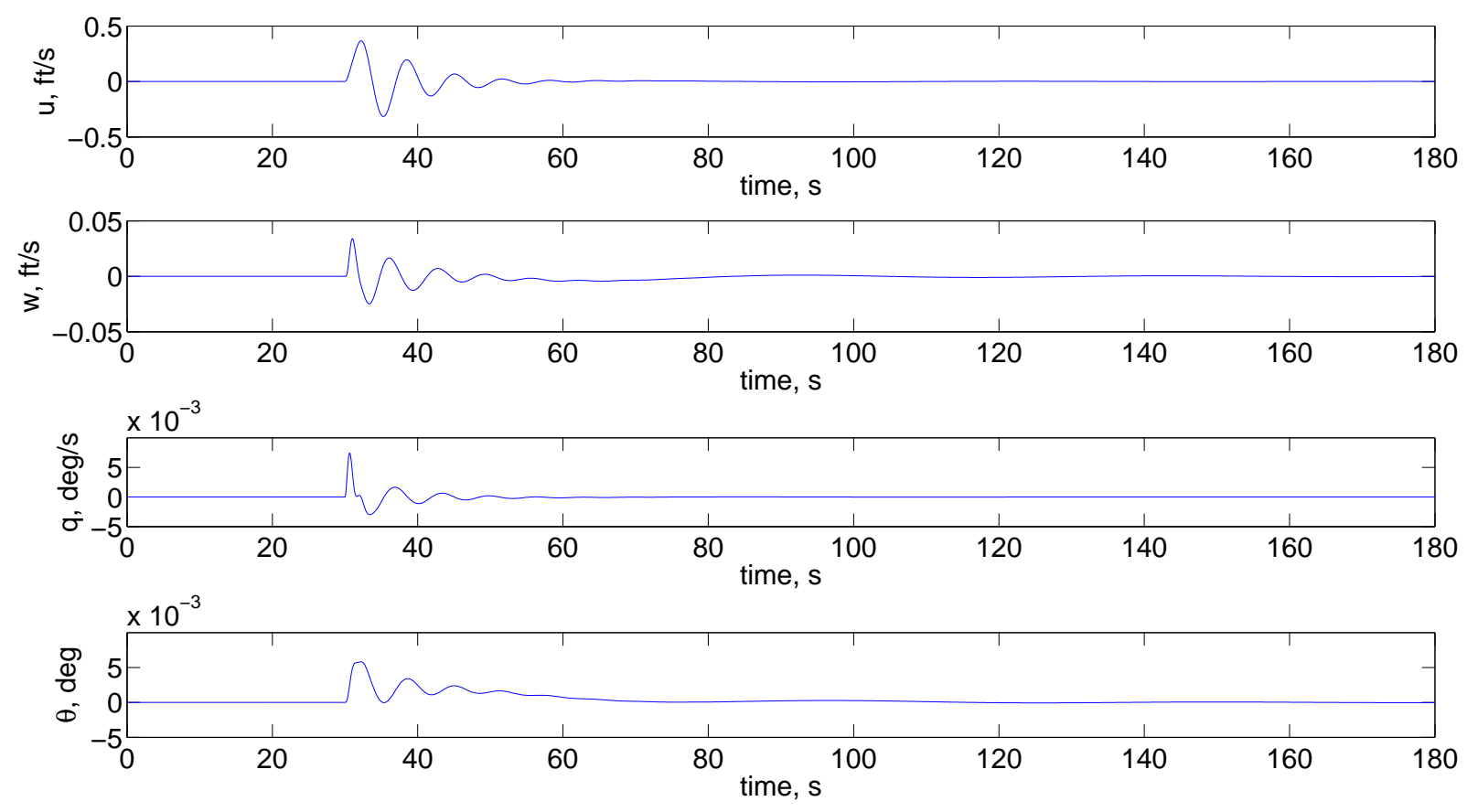

Figure 14. System states $x_{1}=u, x_{2}=w, x_{3}=q, x_{4}=\theta$ (Case V). 

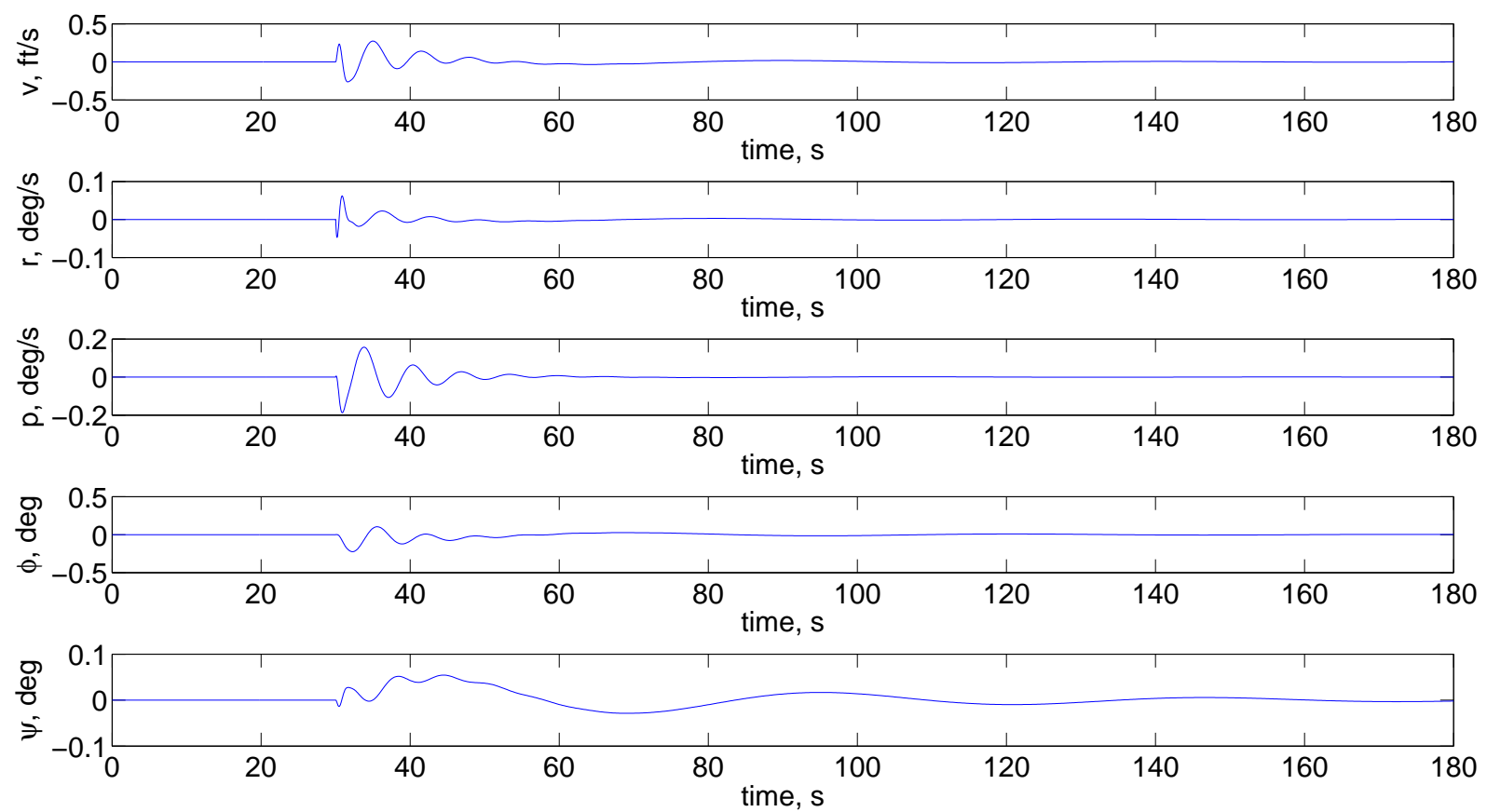

Figure 15. System states $x_{5}=v, x_{6}=r, x_{7}=p, x_{8}=\phi, x_{9}=\psi$ (Case V).
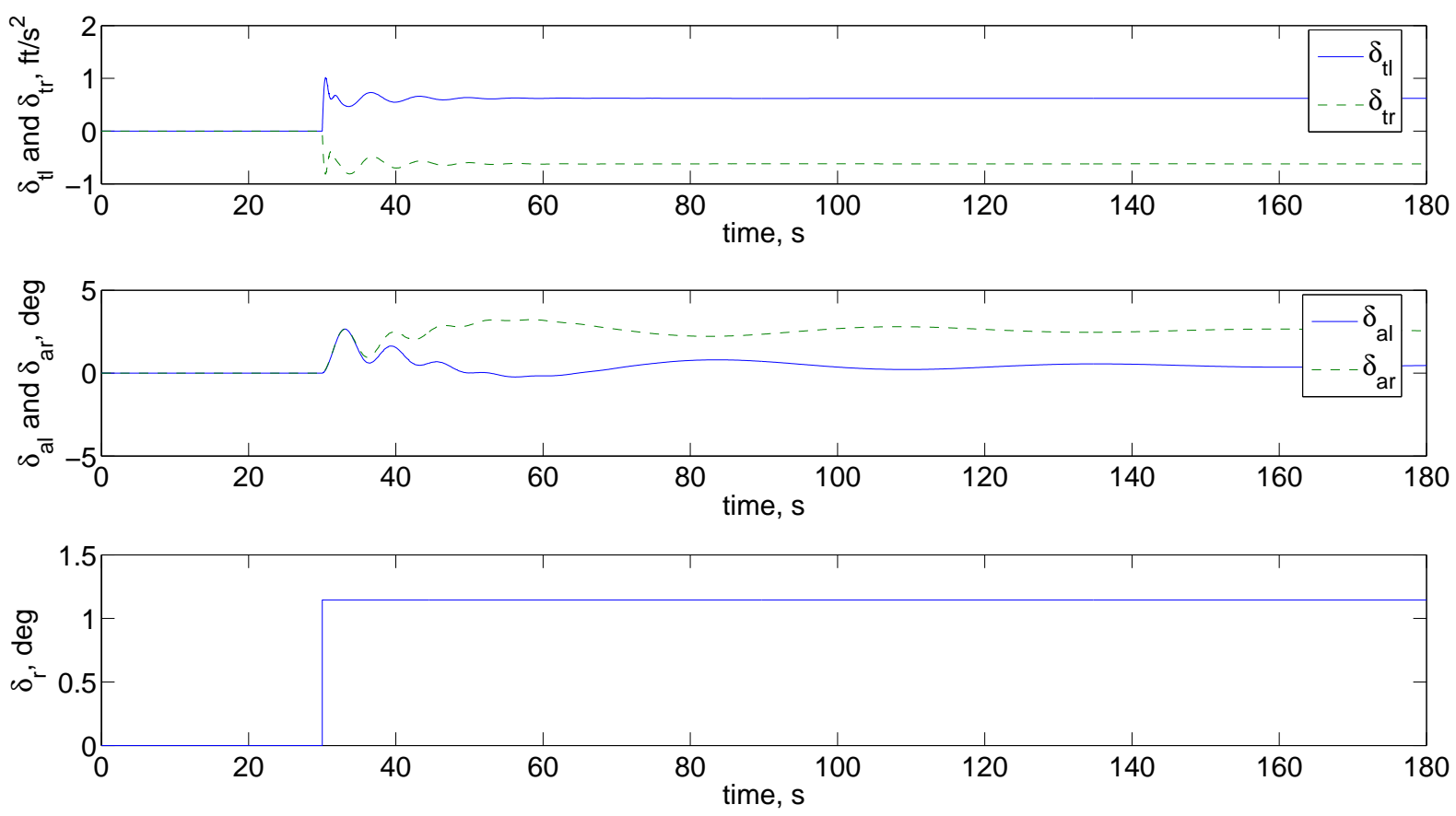

Figure 16. Control signals: $\delta_{t l}, \delta_{t r}, \delta_{a l}, \delta_{a r}$ and $\delta_{r}($ Case V). 

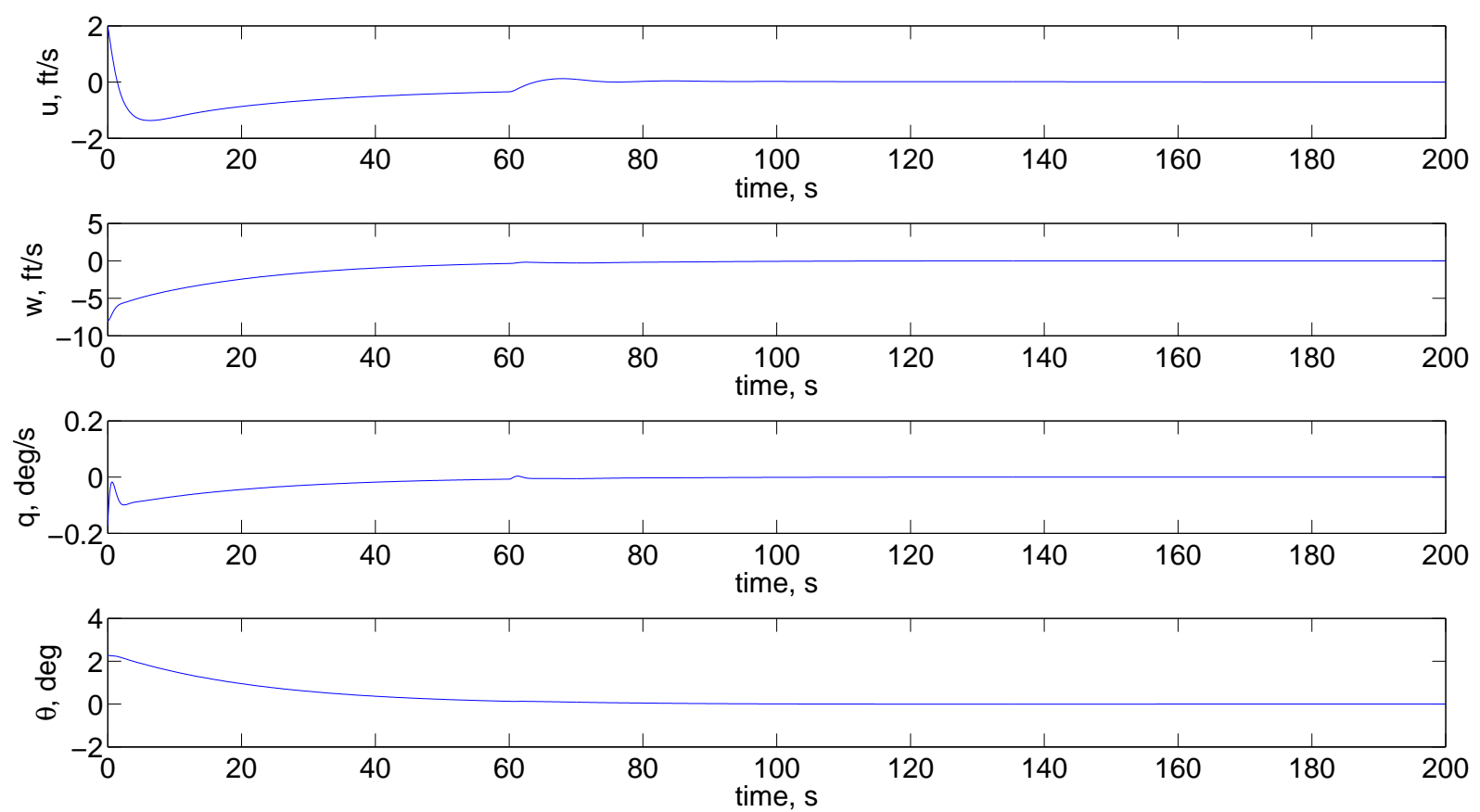

Figure 17. System states $x_{1}=u, x_{2}=w, x_{3}=q, x_{4}=\theta$ (Case VI).
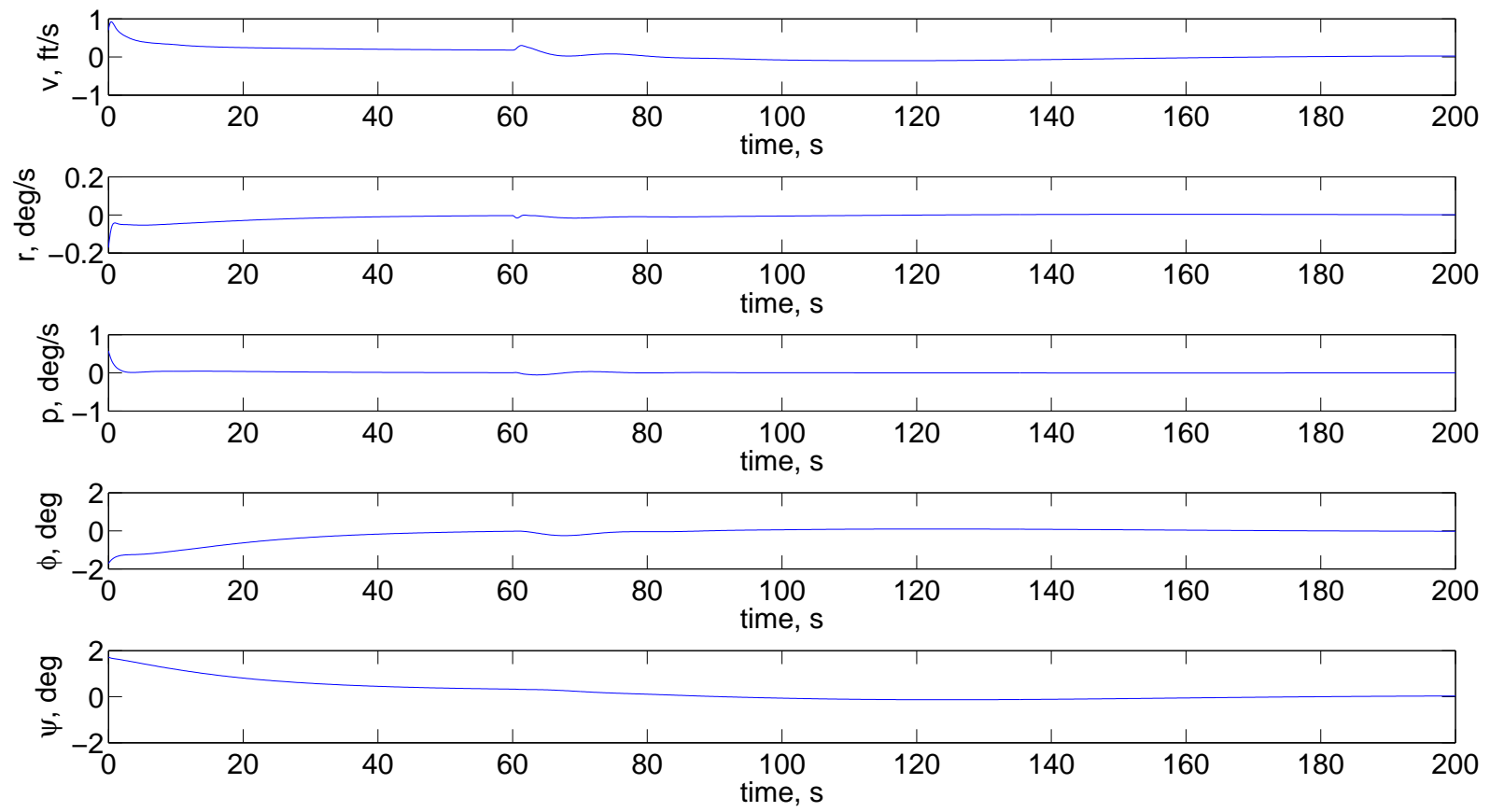

Figure 18. System states $x_{5}=v, x_{6}=r, x_{7}=p, x_{8}=\phi, x_{9}=\psi$ (Case VI). 

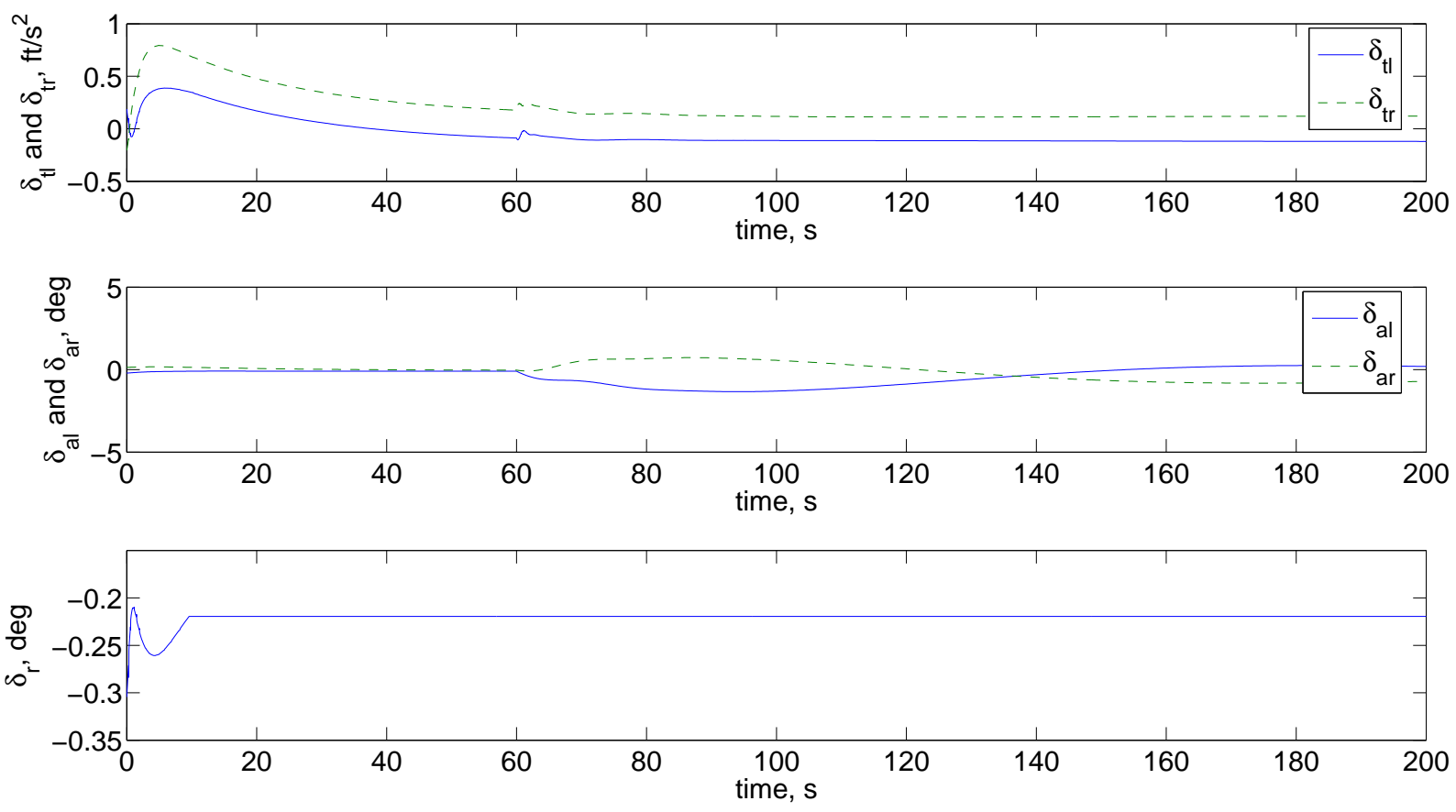

Figure 19. Control signals: $\delta_{t l}, \delta_{t r}, \delta_{a l}, \delta_{a r}$ and $\delta_{r}$ (Case VI).

Case (VI) and (VII), we can see that states are eventually stabilized after switching the adaptive scheme on, which clearly show the effectiveness of the failure compensation scheme comparing with Case (I) and (II).

Case (VIII). In Case (VIII), we consider the aileron failure (49). The failure instant is chosen as $t_{f}=30$ seconds. The initial values for the states are defined in (46). The elements in $\Gamma_{i}(i=1,2, \ldots, 6)$ are chosen as $\left[\begin{array}{lllllllll}0.01 & 0.01 & 0.01 & 0.01 & 0.08 & 0.02 & 0.02 & 4 & 4\end{array}\right]$, and $\lambda_{i}(i=1,2, \ldots, 6)$ are chosen as $\left[\begin{array}{llllll}0.02 & 0.4 & 0.4 & 0.4 & 0.4 & 0.03\end{array}\right]$. The system response and some control inputs are shown in Figures 23, 24 and 25. We can see from the results that all the states can be stabilized with adaptive failure compensation in the presence of aileron failure.

Remark In our simulation, the aircraft is flying in an unsymmetric mode from the very beginning, before the failure occurs. Therefore the engine differentials and two ailerons all participate in the system regulation when the initial values of the states are nonzero even though failures have not occured at that time.

In summary, in our study we simulated some typical aircraft motions for some realistic failure conditions and scenarios in which adaptive compensation of the uncertain rudder and aileron failures is achieved by using the non-failed actuators, primarily engine thrust differentials. The failure uncertainties are characterized by the failure value and failure time instant, both of which are unknown to the adaptive controller. The controller ensures desired system performance under both normal and upset conditions, which cannot be achieved by a fixed controller.

\section{Conclusions and Future Work}

This paper addressed adaptive flight control of aircraft in the presence of rudder and aileron failures, using engine thrust differentials as primary actuators. A dynamic model of aircraft with nonsymmetric controls, i.e., independent engine thrusts, was developed and linearized at certain steady-state operating conditions. An adaptive actuator failure compensation scheme that ensures asymptotic regulation of the aircraft state vector and signal boundedness of the closed-loop system was developed. The scheme was applied for adaptive compensation of a large transport aircraft model under unknown rudder and aileron failure conditions occurring at unknown instants of time. Several simulation results were presented, which show that the functioning actuators, primarily the differential engine thrusts, seamlessly take over for the 

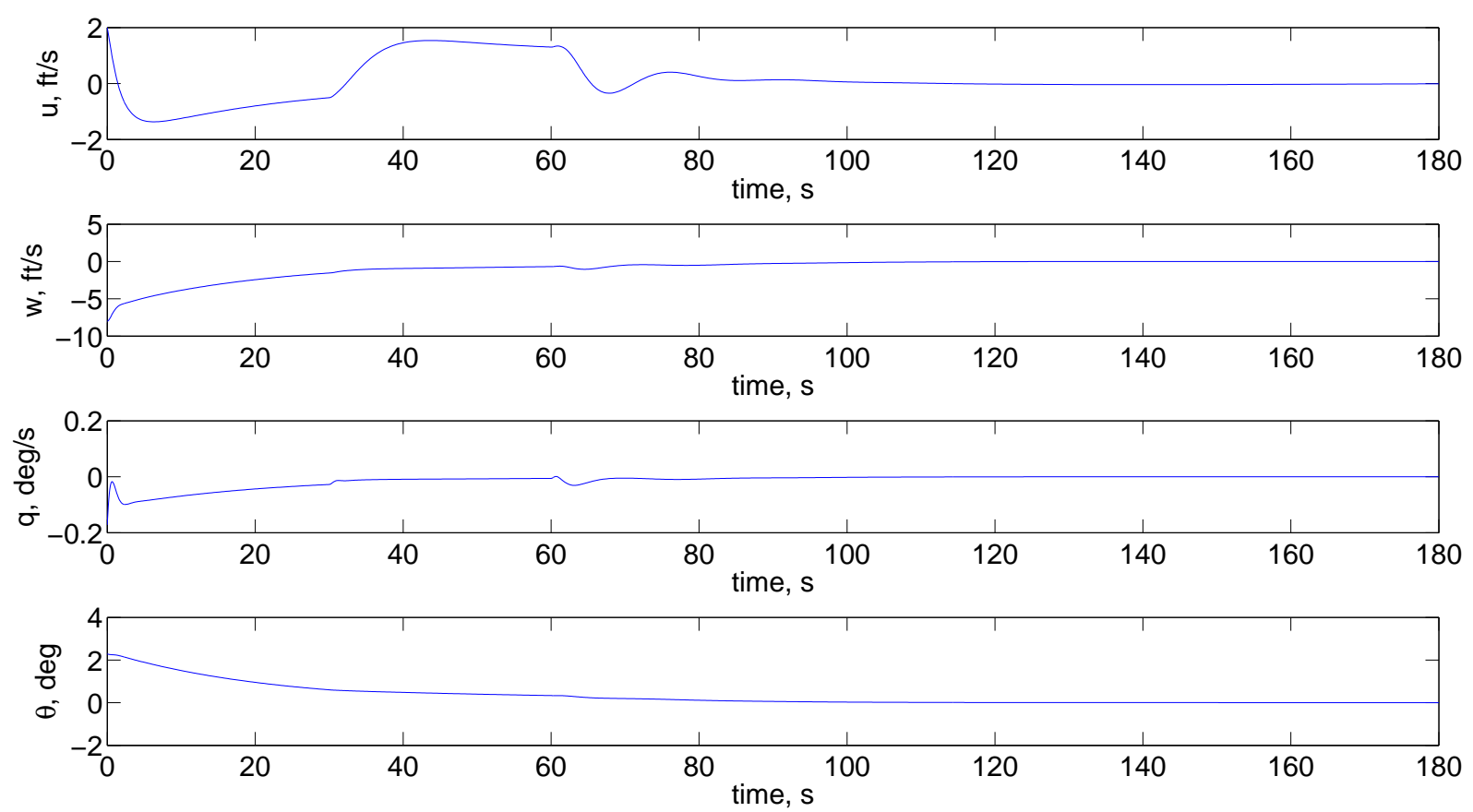

Figure 20. System states $x_{1}=u, x_{2}=w, x_{3}=q, x_{4}=\theta$ (Case VII).
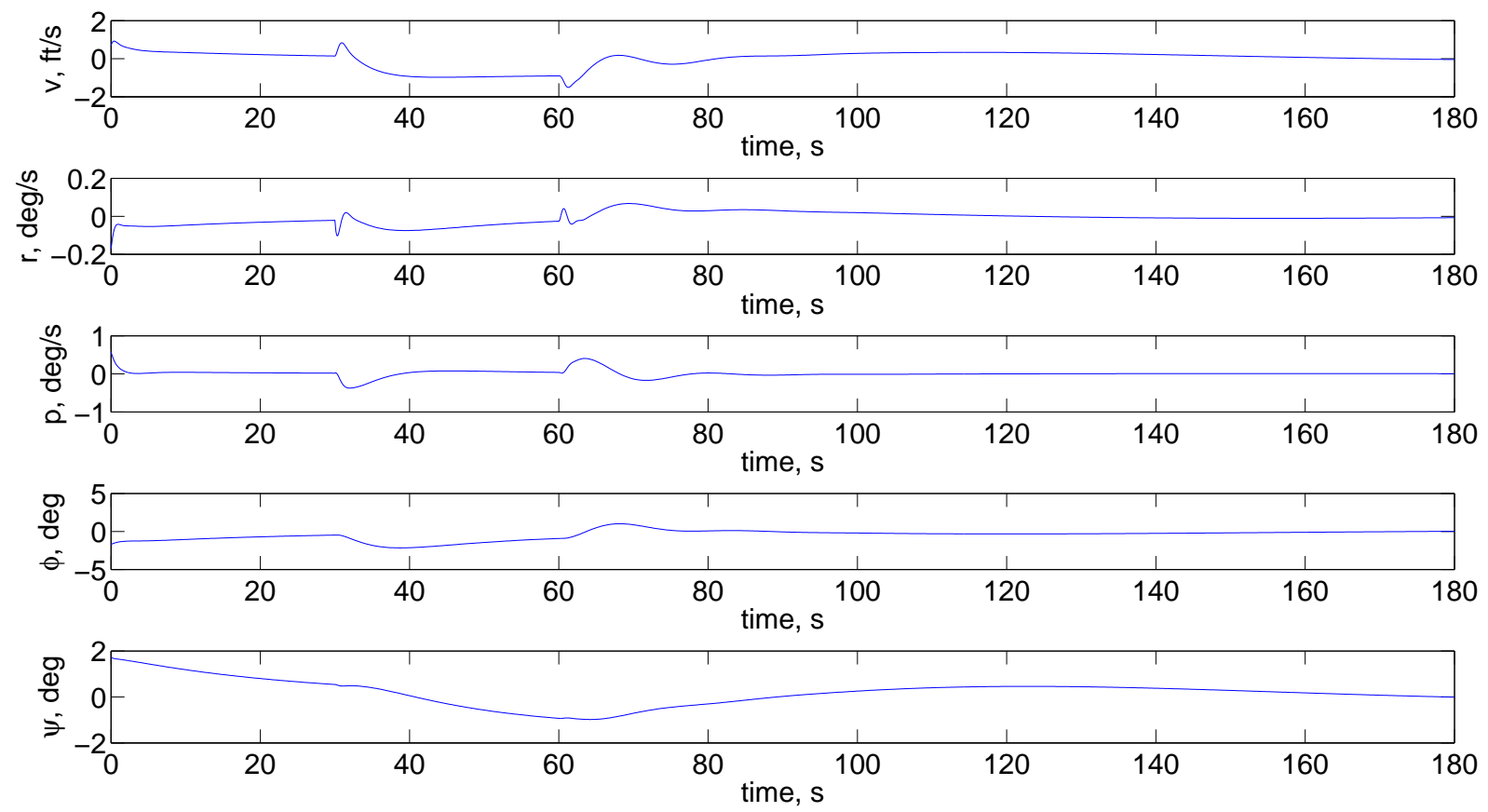

Figure 21. System states $x_{5}=v, x_{6}=r, x_{7}=p, x_{8}=\phi, x_{9}=\psi$ (Case VII). 

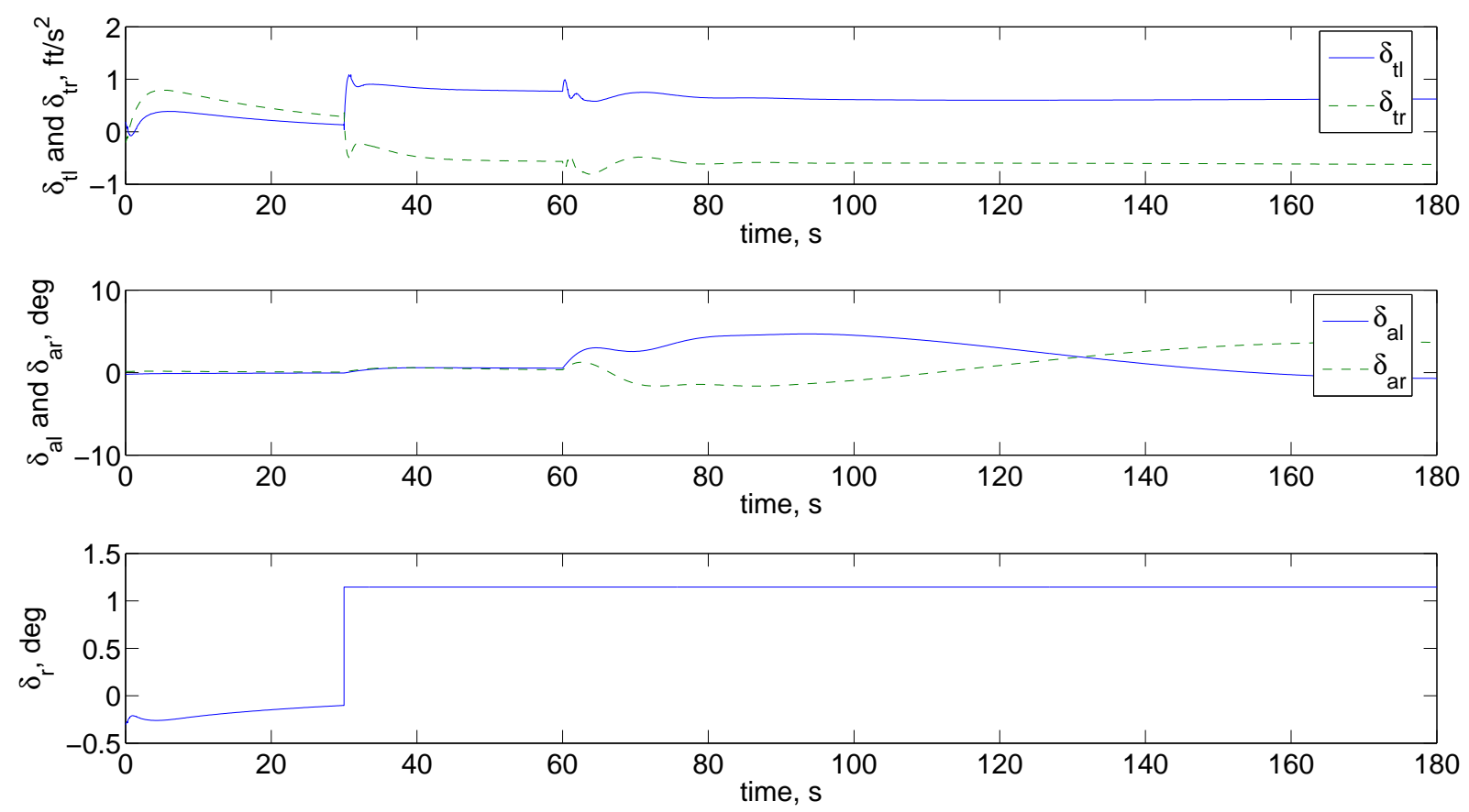

Figure 22. Control signals: $\delta_{t l}, \delta_{t r}, \delta_{a l}, \delta_{a r}$ and $\delta_{r}$ (Case VII).
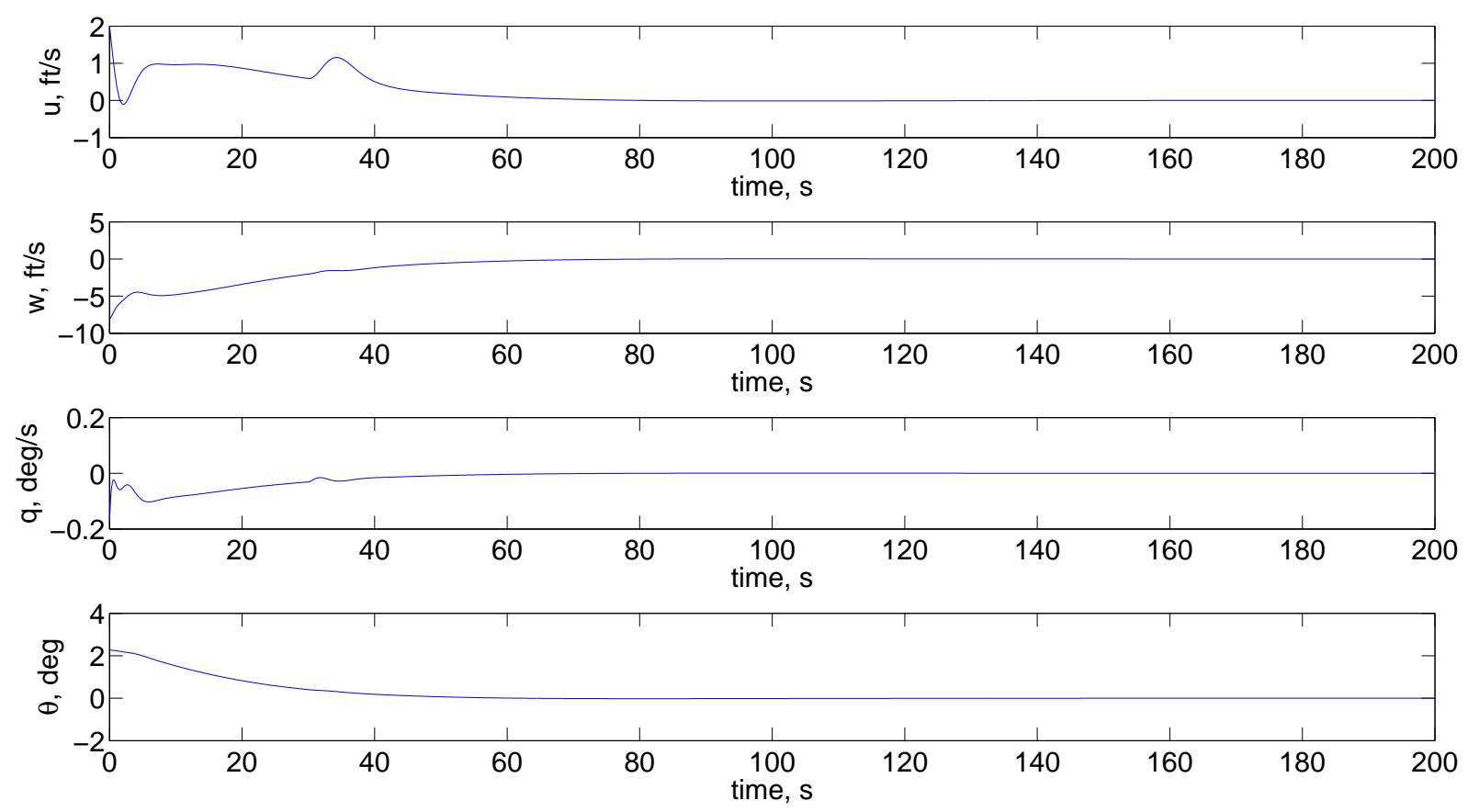

Figure 23. System states $x_{1}=u, x_{2}=w, x_{3}=q, x_{4}=\theta$ (Case VIII). 

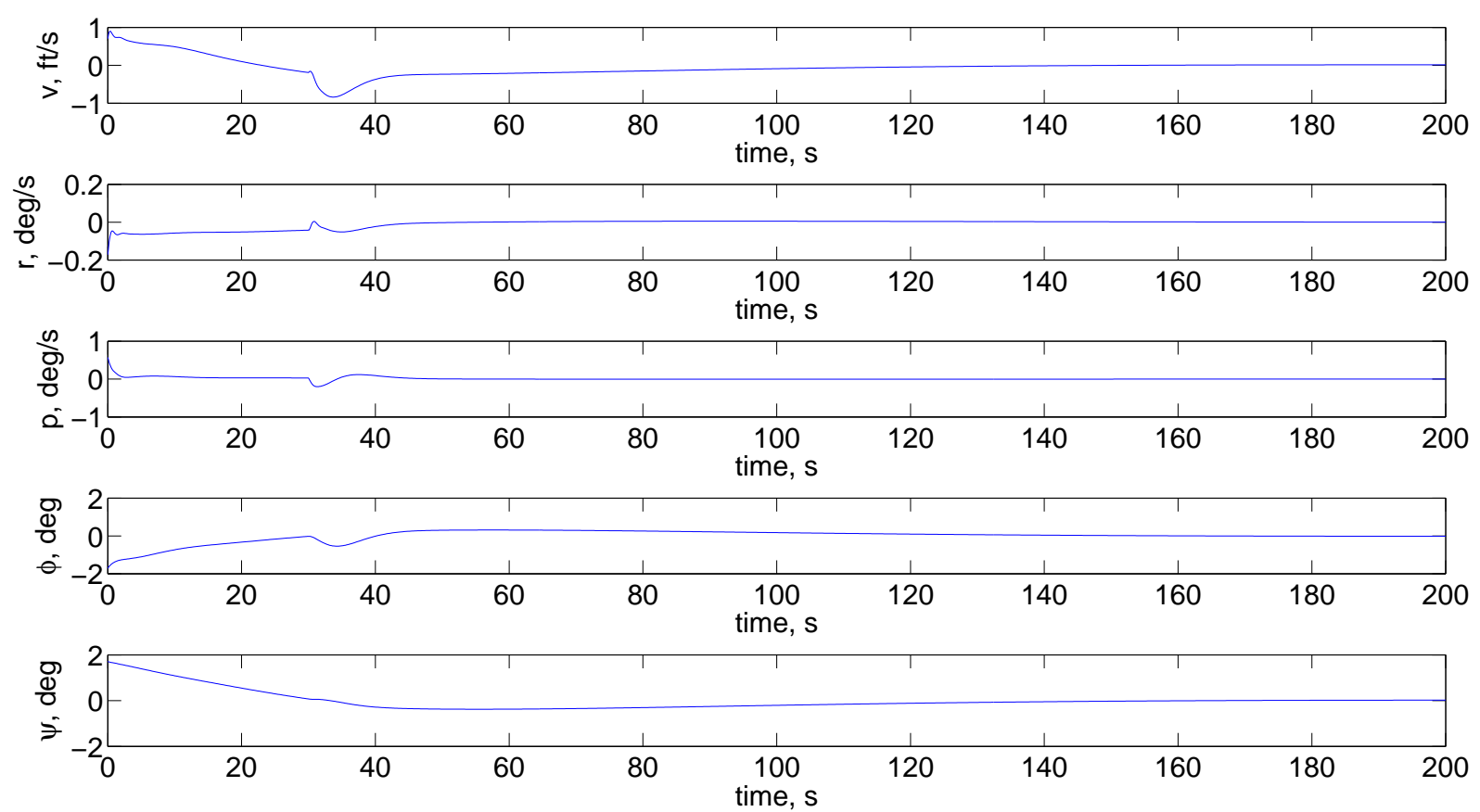

Figure 24. System states $x_{5}=v, x_{6}=r, x_{7}=p, x_{8}=\phi, x_{9}=\psi$ (Case VIII).
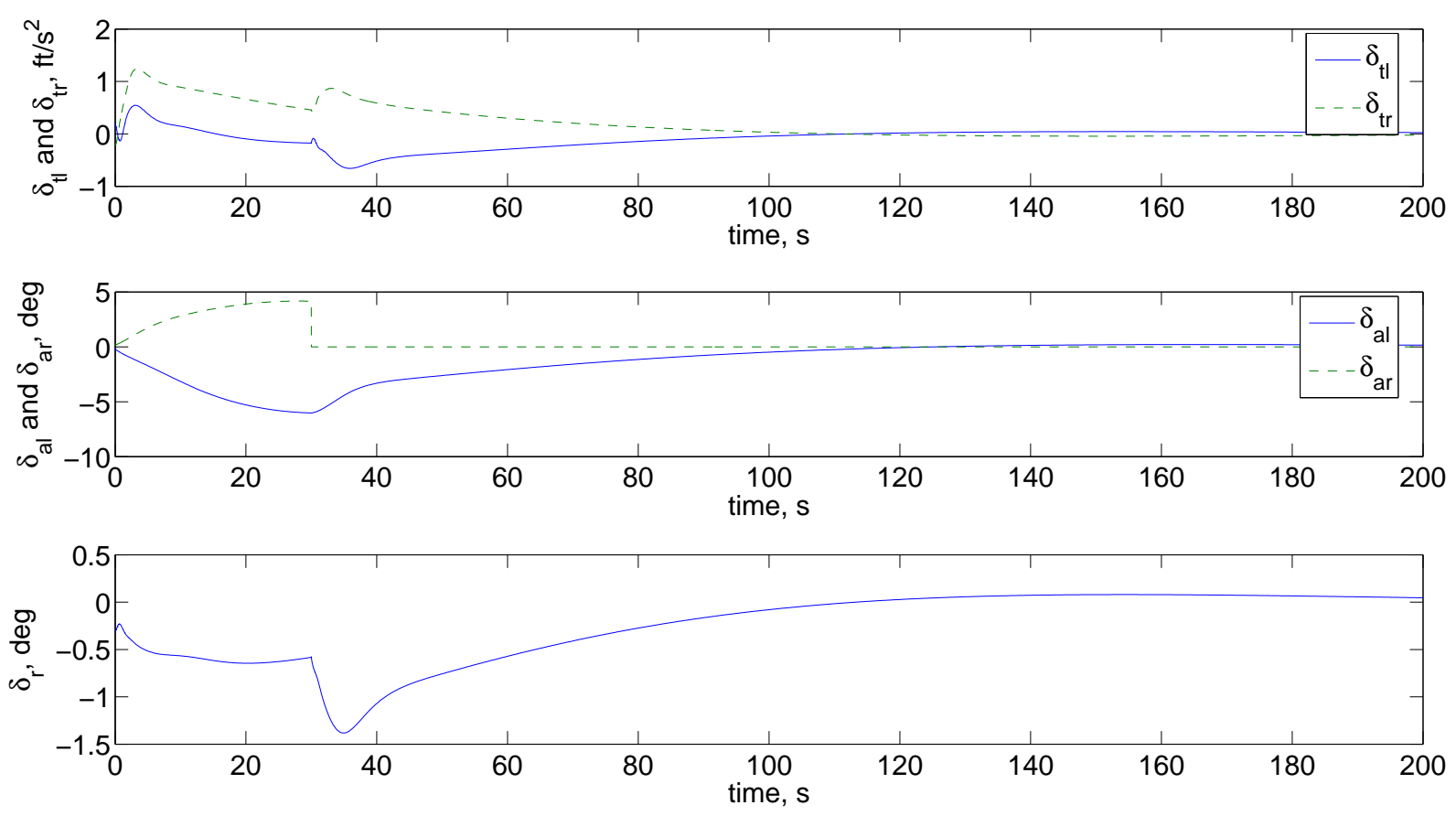

Figure 25. Control signals: $\delta_{t l}, \delta_{t r}, \delta_{a l}, \delta_{a r}$ and $\delta_{r}$ (Case VIII). 
failed rudder or aileron, and demonstrate the effectiveness of the adaptive scheme. The distinguishing feature of this actuator failure compensation scheme, compared to the recent work ${ }^{11}$ by the authors, is the relaxation of the "proportional actuation" condition that requires multiple actuators of the same type and physical characteristics, e.g., multiple rudder segments. It expands the adaptive failure compensation approach to handle the important case where the functioning actuators are of different type from the failed ones. Further research is needed in many aspects of this work. In order to assess the adaptive scheme performance it will be desirable to investigate application to detailed nonlinear piloted aircraft simulations, and subsequently flight testing, possibly on small remotely piloted aircraft. In addition, the adaptive scheme needs to be extended to address unknown damage (in addition to failures) to control actuators, surfaces, engines, and aircraft structure, and also to nonlinear flight regimes.

\section{References}

${ }^{1}$ Haynes, A., The Crash of United Flight 232, Excerpt from talk at NASA Dryden Flight Research Center, 1991.

${ }^{2}$ National Transportation Safety Board, "Aircraft Accident Report: United Airlines Flight 232, McDonnell Douglas DC10-10, Sioux Gateway Airport, Sioux City, Iowa, July 19, 1989," Tech. Rep. NTSB/AAR-90/06, National Transportation Safety Board, 1990.

${ }^{3}$ Tucker, T., Touchdown: The Development of Propulsion Controlled Aircraft at NASA Dryden, No. 16 in Monographs in Aerospace History, NASA History Office, 1999.

${ }^{4}$ Burken, J. J., Maine, T. A., Burcham, Jr., F. W., and Kahler, J. A., "Longitudinal emergency control system using thrust modulation demonstrated on an MD-11 airplane," Proc. of the 32nd AIAA/ASME/SAE/ASEE Joint Propulsion Conference, Lake Buena Vista, Florida, 1996.

${ }^{5}$ Burken, J. J. and Burcham, Jr., F. W., "Flight-test results of propulsion-only emergency control system on MD-11 airplane," Journal of Guidance, Control and Dynamics, Vol. 20, No. 5, 1997, pp. 980-987.

${ }^{6}$ Jonckheere, E. A., Yu, G. R., and Chiang, C. Y., " $H_{\infty}$ control of crippled aircraft in lateral motion with throttles only," Proc. of the 35th Conference on Decision and Control, Kobe, Japan, 1996.

${ }^{7}$ Bryson, Jr., A. E., Control of Spacecraft and Aircraft, Princeton University Press, NJ, 1994. 2002.

${ }^{8}$ Franklin, G. F., Powell, J. D., and Emami-Naeini, A., Feedback Control of Dynamic Systems, Prentice-Hall, NJ, 4th ed.,

${ }^{9}$ Stevens, B. L. and Lewis, F. L., Aircraft Control and Simulation, John Wiley \& Sons, NY, 1992.

${ }^{10}$ Rugh, W. J., Linear System Theory, Prentice-Hall, NJ, 2nd ed., 1996.

${ }^{11}$ Tao, G., Chen, S., Tang, X., and Joshi, S. M., Adaptive Control of Systems with Actuator Failures, Sringer-Verlag, London, 2004.

${ }^{12}$ Tang, X., Tao, G., Wang, L., and Stankovic, J. A., "Robust and adaptive actuator failure compensation designs for a rocket fairing structural-acoustic model," IEEE Transaction on Aerospace and Electronic Systems, Vol. 40, No. 4, October 2004, pp. 1359-1366.

${ }^{13}$ Lewis, F. L., Applied Optimal Control and Estimation, Prentice-Hall, NJ, 1992. 